\title{
A phenomenological explanation for the anomalous ion heating observed in the JET alpha-heating experiment of 1997
}

\author{
D. Testa and M. Albergante \\ CRPP, Association EURATOM-Confédération Suisse, EPFL, Lausanne, Switzerland
}

Received 19 October 2011, accepted for publication 12 June 2012

Published 11 July 2012

Online at stacks.iop.org/NF/52/083010

\begin{abstract}
In the so-called 'alpha-heating' experiment performed on the JET tokamak during the deuterium-tritium campaign of 1997, the ion temperature was found to be far exceeding (both in absolute value and in its rise time) the level that could have been expected from direct collisional heating by the fusion-born alpha particles themselves and energy equipartition with the electrons. To date, no explanation has been put forward for this long standing puzzle, despite much work having been performed on this subject in the early 2000s.

Two analysis methods that have recently become available have been employed to re-analyse these observations of an anomalous ion heating. First, an algorithm based on the sparse representation of signals has been used to analyse magnetic, reflectometry and electron-cyclotron emission measurements of the turbulence spectra in the drift-wave range of frequencies. This analysis has then been complemented with turbulence simulations performed with the GENE code.

We find, both experimentally and in the simulations, that the presence of a minority, but sufficiently large, population of fusion-born alpha particles that have not yet fully thermalized stabilizes the turbulence in the ion-drift direction, but practically does not affect the turbulence in the electron-drift direction. We link such stabilization of the ion-drift-wave turbulence to the increase in the ion temperature above the level achieved in similar discharges that did not have (at all or enough) alpha particles. When the fusion-born alpha particles have fully thermalized, the turbulence spectrum in the ion-drift direction reappears at somewhat larger amplitudes, which we link to the ensuing reduction in the ion temperature. This phenomenological dynamics fully corresponds to the actual experimental observations. By taking into account an effect of the alpha particles that had not been previously considered, our new analysis finally presents a phenomenological explanation for the so-far-unexplained anomalous ion heating observed in the JET alpha-heating experiment of 1997.

Through the formulation of an empirical criterion for ion-drift-wave turbulence stabilization by fusion-born alpha particles, we also show why similar observations were not made in the other deuterium-tritium experiments run so far in JET and TFTR. This allows assessing the operational domain for this stabilization mechanism for ion-drift-wave turbulence in future burning plasma experiments such as ITER, which may open a new path towards the sustainment of a high energy gain in such forthcoming devices.
\end{abstract}

(Some figures may appear in colour only in the online journal)

\section{Introduction}

One of the main aims of the JET Deuterium-Tritium Experiment (DTE1) in 1997 [1] was to verify the bulk plasma heating by the fusion-born alpha particles $(\alpha \mathrm{s})$ [2]. Since the birth energy of fusion-born $\alpha \mathrm{s}$ is $3.5 \mathrm{MeV}$, in excess of $85 \%$ of their energy was expected to be transferred to the bulk plasma electrons directly through collisions over the time scale of the slowing-down time of the $\alpha \mathrm{s}\left(\tau_{\alpha \mathrm{e}}\right)$, where, in the core of those plasmas, $\tau_{\alpha \mathrm{e}} \sim 1.2 \mathrm{~s}$ is of the same order of the energy confinement time $\tau_{E}, \tau_{E} \sim 0.7 \mathrm{~s}$ to $\tau_{E} \sim 1 \mathrm{~s}$. The corresponding prediction of an increase over the same time scale in the electron temperature $T_{\mathrm{e}}$ as a function of the DT mixture ratio, hence the total fusion power, and the alphafusion power and concentration, was fully verified during the DTE1 campaign [3]. At that time, it was noted that the increase in the ion temperature $T_{\mathrm{i}}$ was by far exceeding in magnitude and in its rise time $\left(\tau_{\mathrm{Ri}}=\left(\mathrm{d} \log \left(T_{\mathrm{i}}\right) / \mathrm{d} t\right)^{-1} \sim 0.8 \mathrm{~s}\right)$ the value that could have been expected from energy equipartition with the electrons (occurring over a time scale $\tau_{\mathrm{ei}} \sim 5 \mathrm{~s}$ ) or by the residual small $(<15 \%)$ direct collisional heating by the $\alpha$ s themselves on the ions. Note also that the slowing-down 
time of the $\alpha$ s on the background thermal ions $\tau_{\alpha \mathrm{i}} \sim 4 \mathrm{~s}$ was slightly shorter than the energy equipartition time in the DTE1 discharges. Much work was performed in the early 2000s to validate the $T_{\mathrm{i}}$ measurements, and many possibilities were put forward to explain these 'anomalous' ion heating data, but none was found to be able to account for the observed ion temperature increase [4].

In this work we focus on a renewed analysis of the turbulence data obtained in the JET alpha-heating experiment of 1997. There are two main reasons for this analysis. First, there is an historical perspective in that the possibility that a new high-power DT experiment will be carried out in JET in the near future [5] has motivated a renewed interest in the subject of this anomalous ion heating with the $\alpha$ s, since this effectively remains the only outstanding puzzle to be solved from the original DTE1 campaign. Second, as the collisional slowing-down of the fusion-born $\alpha$ s on the electrons and the ensuing energy equipartition with the ions are the essential ingredients for self-sustainment of the fusion reactivity in future burning plasma experiments, it is important to assess whether this observation of a direct, and much faster than expected, ion heating in the presence of $\alpha$ s could be linked to some underlying physics mechanisms that, in turn, could be used to optimize the path to the sustainment of a high-energy gain in forthcoming burning plasma devices such as ITER [6].

For our analysis we start from the ansatz that, under otherwise steady-state plasma conditions, there is a direct link between the electron and ion turbulence in the driftwave range of frequencies and the temporal evolution of the electron and ion temperatures. The turbulence spectra are experimentally evaluated using magnetic and internal measurements (such as electron-cyclotron emission (ECE) and $\mathrm{O}$ - and $\mathrm{X}$-mode reflectometry). These data have been analysed in this work using an innovative algorithm based on the sparse representation of signals (SRSs) [7]. We then have compared the experimental results with the theoretical turbulence predictions obtained using the GENE code [8]. This approach allows us to analyse the energy-dependent effect of a minority population on the predicted turbulence spectrum as a function of the $\mathrm{D} / \mathrm{T}$ mixture ratio and the equivalent temperature of the alpha particle population.

It is important to note at this point that neither the SRS method nor the GENE code (and actually even the ideas behind these tools) was available neither at the time of the first analysis of the DTE1 data in 1997-1998 nor at the time of the subsequent dedicated analysis of the anomalous ion heating data in the early 2000s. The analysis of the turbulence measurements reported in [4] was performed using a fast Fourier transform (FFT) in the time versus frequency domain, and phase fitting and singular value decomposition (SVD) techniques in the toroidal angle $(\phi)$ versus toroidal mode number $(n)$ domain. In particular, use of SVD-type algorithms at that time suffered severe numerical limitations due to the available CPU and RAM resources, as the analysis of incoherent turbulence requires a substantially larger number of topos and cronos than that needed for the analysis of coherent modes. Phase fitting did not suffer from these numerical limitations, but it was (and still is) unable to de-convolve a spectrum made up of different components whose number and amplitude ratio is unknown a priori.
A short overview of some of the main findings of our studies has been presented elsewhere $[9,10]$. Here we expand on these earlier presentations by developing the analysis of the measurements and the GENE modelling of the turbulence in the ion and electron drift-wave channels, by formulating an empirical criterion for ion-drift-wave turbulence stabilization by fusion-born $\alpha$ s that allows us to provide some qualitative explanation of why such anomalous ion heating had not been observed in other previous DT experiments, and by evaluating the possibility of applying the results of these studies to future DT experiments in burning plasma tokamaks such as ITER.

This paper is organized as follows. In section 2, we briefly review the experimental observations for the anomalous ion heating that were extensively reported in [4] with the purpose of facilitating the reading of this work. In section 3 we present the results of the turbulence simulations performed with the GENE code. In section 4 we show the measurements of the drift-wave turbulence in the ion and electron channels at selected time points for all the discharges that were used for the alpha-heating experiment, and their analysis using the SRS method (for which the basic mathematical formulation is presented in the appendix to facilitate the reading of this work). In section 5 we then provide a simple, qualitative explanation as to why this anomalous ion heating was not observed in other DT experiments. In section 6 we show the results of initial simulations performed for the ITER reference steady-state scenario using the ion temperature gradient turbulence suppression mechanism presented here. Finally, section 7 presents the conclusions of this work and puts forward suggestions for dedicated measurements that could be performed in future high-power DT experiments in JET so as to further validate the phenomenological explanation for the anomalous ion heating with $\alpha$ s that is proposed in this work, hence improving and providing quantitative predictions for ITER.

\section{The anomalous ion heating observed in the JET DTE1 experiment}

The DTE1 alpha-heating experiment scanned the DT mixture ratio $n_{\mathrm{T}} /\left(n_{\mathrm{D}}+n_{\mathrm{T}}\right)$, where $n_{\mathrm{D}}$ and $n_{\mathrm{T}}$ are the deuterium and tritium densities, respectively, in the range $0 \leqslant n_{\mathrm{T}} /\left(n_{\mathrm{D}}+n_{\mathrm{T}}\right) \leqslant$ 0.92 in a hot ion H-mode [11] with plasma current $I_{\mathrm{p}}=3.8 \mathrm{MA}$ and on-axis toroidal magnetic field $B_{\phi}=3.4 \mathrm{~T}$, while keeping constant the magnetic equilibrium, the current profile and the background plasma density. All of these discharges were additionally heated using only neutral beam injection (NBI), with constant NBI power $P_{\mathrm{NBI}}=10.5 \mathrm{MW}$, this value having been selected so as to keep a constant ion temperature by matching the predicted ion heat losses, thus facilitating the evaluation of the direct alpha-heating to the electrons. The NBI ions were injected using the same fuel mixture as the DT ratio of the background plasma, at the energies required to maximize direct collisional heating to the thermal ions (140 keV for D-NBI, $160 \mathrm{keV}$ for T-NBI ions), as for these discharges the collision frequency of the high-energy NBI ions with the electrons equals that with the background thermal ions at $E_{\mathrm{D}, \mathrm{CRIT}} \sim 150 \mathrm{keV}$ and $E_{\mathrm{T}, \mathrm{CRIT}} \sim 240 \mathrm{keV}$ in the plasma core, respectively. Here $E_{\mathrm{X}, \mathrm{CRIT}}[12]$ is the energy at which the collision frequency of the non-thermal NBI ion species $\mathrm{X}$ 
with thermal electrons $\left(1 / \tau_{\mathrm{Xe}}\right)$ equals that with thermal ions $\left(1 / \tau_{X i}\right)$.

In a series of comparison discharges, ion cyclotron resonance frequency (ICRF) heating was used to replace the fusion-born and velocity-space isotropic $\alpha$ s with still MeV-energy, but velocity-space anisotropic fast hydrogen and deuterium ions $[3,4]$. When the DT mixture ratio was in the range $0.45<n_{\mathrm{T}} /\left(n_{\mathrm{D}}+n_{\mathrm{T}}\right)<0.65$, a clear maximum in the alpha-fusion power $P_{\alpha \mathrm{FUS}} \sim 1.3 \mathrm{MW}$ was observed together with a corresponding increase in the plasma thermal energy content $\Delta W_{\mathrm{TH}} \sim 1 \mathrm{MJ}$ and electron temperature in the plasma core $\Delta T_{\mathrm{e} 0} \sim 1.3 \mathrm{keV}$. The initial analyses of this set of discharges concentrated on the electron heating aspects, and particularly on the dependence of the electron temperature on the DT mixture ratio and alpha-fusion power [3]. The values of $\Delta W_{\mathrm{TH}}$ and $\Delta T_{\mathrm{e} 0}$ were found to be in accordance with the predictions of in excess of $85 \%$ of the alpha-energy being collisionally transferred to the electrons.

However, the observed increase in the core ion temperature, $\Delta T_{\mathrm{i} 0} \sim 3 \mathrm{keV}$, was by far exceeding the value that could have been expected from energy equipartition with the electrons or by the residual (not exceeding 15\%) direct collisional alpha-heating to the ions [4]. The ion heating with the $\alpha$ s was observed to be approximately three times that of the electrons, and was occurring on time scales much faster than those set by energy equipartition with the electrons. This level of ion heating was not observed when MeV-energy, but anisotropic, ions produced by ICRF were substituted to the fusion-born and isotropic $\alpha \mathrm{s}$ in D-only plasmas, although these plasmas showed a very similar level of electron heating, thus indicating a possible isotopic effect of the DT fuel mixture and a direct, but unaccounted and totally unexpected, role of the $\alpha$ s themselves.

This increase in $T_{\mathrm{i} 0}$ was so unexpected and puzzling (as effectively all the other predictions on the alpha-heating were indeed quantitatively confirmed by the measurements obtained during the DTE1 campaign) that much care was taken in a subsequent analysis of the $T_{\mathrm{i}}$ data obtained using charge-exchange spectroscopy measurements and the ensuing transport analyses that were performed using the TRANSP [13] and JETTO [14] codes. These analyses (see the issue of Nuclear Fusion devoted to the JET DTE1 experiments [15], and also [16]) showed that using the measured $T_{\mathrm{i}}$ profiles for all the discharges considered in the alpha-heating experiment produces a very good agreement between the measured and the predicted neutron rate and plasma thermal energy content. Diagnostic inconsistencies on the $T_{\mathrm{i}}$ measurements could then be ruled out. Following earlier results from the Tokamak Fusion Test Reactor (TFTR) [17], an isotopic effect on the energy confinement, heat transport and electron temperature was also considered [18-23]. However, since the $T_{\mathrm{i}}$ and $T_{\mathrm{e}}$ increase was measured to be similar and not significant at the two extremes of the DT mixture ratio scan $\left(n_{\mathrm{T}} /\left(n_{\mathrm{D}}+n_{\mathrm{T}}\right)=\right.$ 0 , as in the comparison discharges with ICRF heating, and $\left.n_{\mathrm{T}} /\left(n_{\mathrm{D}}+n_{\mathrm{T}}\right)=0.92\right)$, possible isotopic effects in the energy confinement and electron-ion equipartition had to also be excluded as the dominant reason for the observed $T_{\mathrm{i} 0}$ increase. Finally, since for the case with $n_{\mathrm{T}} /\left(n_{\mathrm{D}}+n_{\mathrm{T}}\right) \sim 0.6$ the ion thermal conductivity $\chi_{\mathrm{i}}$ calculated by TRANSP was found to be reduced by a factor $\sim 2$ in the core plasma region
Table 1. Main parameters for the set of discharges that constituted the alpha-heating experiment: $n_{\alpha}$ is the alpha particle density and the brackets ' $\langle X\rangle$ ' indicate that $X$ is a volume-averaged quantity.

\begin{tabular}{lllll}
\hline $\begin{array}{l}\text { Discharge } \\
\text { Number }\end{array}$ & $n_{\mathrm{T}} /\left(n_{\mathrm{D}}+n_{\mathrm{T}}\right)$ & $\begin{array}{l}\left\langle n_{\alpha} / n_{\mathrm{e}}\right\rangle \\
(\%)\end{array}$ & $\begin{array}{l}\text { Time-point } \\
\# 1(\mathrm{~T} 1)\end{array}$ & $\begin{array}{l}\text { Time-point } \\
\# 2(\mathrm{~T} 2)\end{array}$ \\
\hline$\# 40365$ & 0.00 & 0.00 & 13.13 & 14.40 \\
$\# 41069$ & 0.00 & 0.00 & 13.53 & 14.25 \\
$\# 42870$ & 0.19 & 1.34 & 13.14 & 14.50 \\
$\# 42856$ & 0.53 & 3.52 & 13.14 & 14.10 \\
$\# 42847$ & 0.60 & 3.47 & 13.13 & 14.20 \\
$\# 42840$ & 0.78 & 2.74 & 13.14 & 14.20 \\
$\# 43011$ & 0.92 & 1.07 & 13.13 & 14.00 \\
$\# 42940$ & 0.21 & 1.12 & 13.15 & 14.00 \\
$\# 43491$ & 0.00 & 0.00 & 13.15 & 14.00 \\
\hline
\end{tabular}

$0.3<r / a<0.7$ (where $a$ is the plasma minor radius and $r$ is the radial coordinate along the plasma midplane), this anomalous ion heating was then empirically attributed to a direct, but unexplained and totally unexpected, effect of the $\alpha \mathrm{s}$ on $\chi_{\mathrm{i}}$. The observation of this anomalous ion heating during the DTE1 experiment has then remained a puzzle until now.

Table 1 gives the main parameters for the set of discharges that constituted the alpha-heating scan, and similarly figures 1 and 2 show the main plasma parameters for one of these discharges (\#42856), with the aim of providing a basic and simply illustrative overview of the background plasma data in this experiment. The readers are referred to $[1-4,11,15,16]$ for an exhaustive and detailed overview of the measurements and ensuing theoretical and modelling analyses performed for the DTE1 campaign in general and for the alpha-heating experiment specifically.

As shown in table 1, two different time points are selected for the turbulence analysis that will be reported in section 4 of this work. The first time-point (T1) corresponds to the early NBI heating phase, i.e. within a fraction of one slowing downtime of the $\alpha \mathrm{s}$ on the electrons ( $\tau_{\alpha \mathrm{e}} \sim 1.2 \mathrm{~s}$ at the $\alpha \mathrm{s}$ birth energy of $3.5 \mathrm{MeV}$ ) from the time when the $P_{\mathrm{NBI}}=10.5 \mathrm{MW}$ flat-top has been reached, therefore much before the time-point corresponding to the peak alpha-fusion power. The second time-point (T2) for the analysis is selected at the time-point of maximum $T_{\mathrm{e} 0}$, which follows within approximately one $\tau_{\alpha \mathrm{e}}$, the time-point of the peak $P_{\alpha \mathrm{FUS}}$ value, i.e. when there is already a large fraction of fusion-born $\alpha$ s that have had the time to fully thermalize.

Figure 1 shows the main background plasma parameters for \#42856 over the time window of interest, corresponding to the NBI heating phase. For illustrative purposes, only a few time points have been selected from the complete time traces shown in $[1-4,15,16]$, and the original data were also binned over $\pm 25 \mathrm{~ms}$ for graphical purposes. (Again for graphical purposes, this procedure was also employed for the data presented in figures $2(a),(b)$ and 3.) Note that here (as well as in the following) the quantity $n_{\mathrm{He}_{4}}$ indicates the thermal $\mathrm{He}_{4}$ density, which is made up by fusion-born $\alpha$ s that have had the time to fully thermalize, and by the background (thermal) $\mathrm{He}_{4}$ impurity content, whereas $n_{\alpha}$ indicates the density of fusionborn $\alpha$ s with energy $>3 \mathrm{MeV}$ (i.e. practically corresponding to the density of $\alpha \mathrm{s}$ at birth), which was computed using TRANSP and verified using the measurements obtained in the plasma core with a high-energy neutral particle analyser [24-26]. 


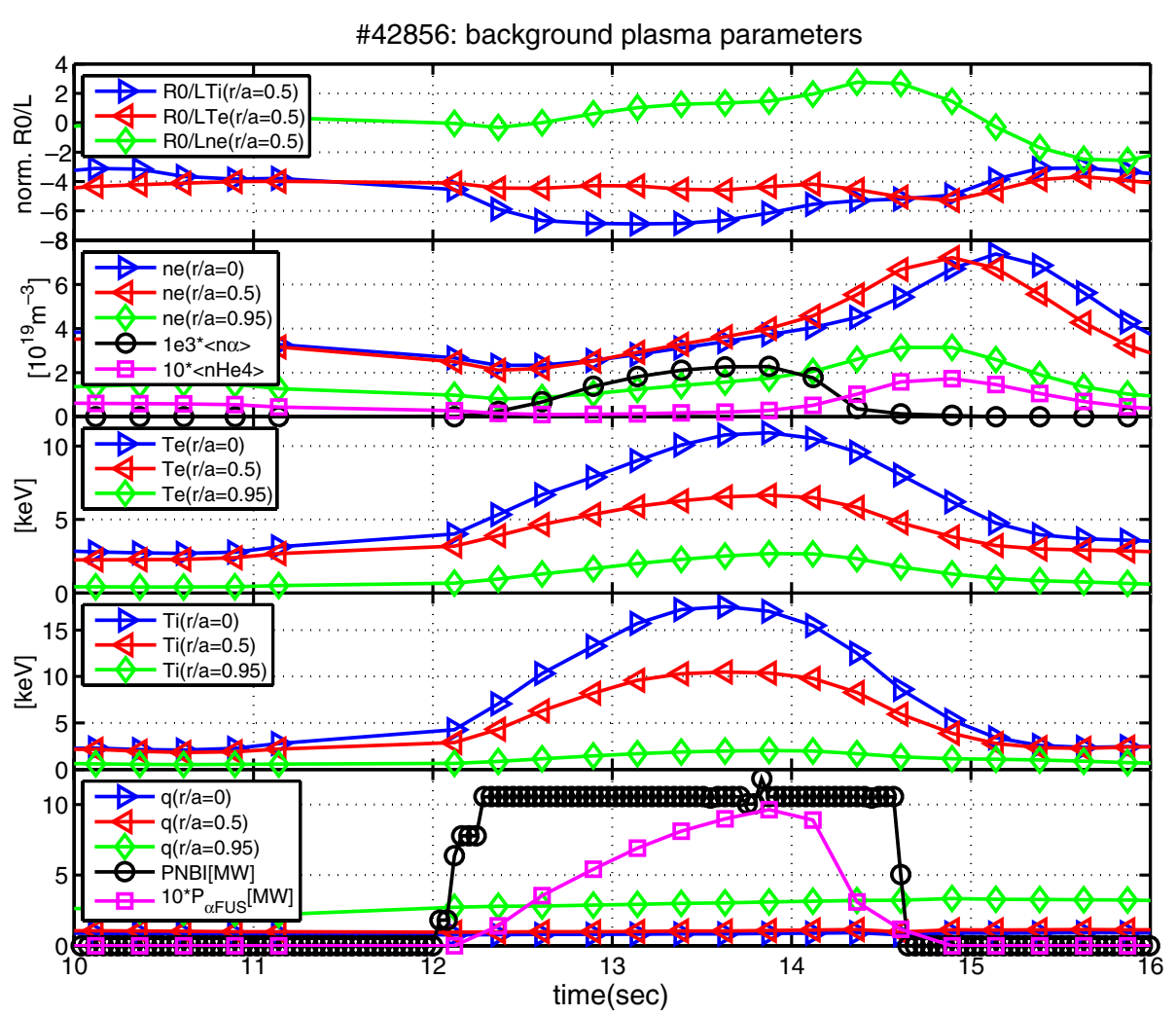

Figure 1. Main background plasma parameters over the time window of interest for \#42856; $q(r)$ is the safety factor profile, $n_{\alpha}$ is the density of fusion-born $\alpha$ s at their birth energy, $n_{\mathrm{He}_{4}}$ is the density of thermal $\mathrm{He}_{4}$ ions (hence including impurity He $\mathrm{H}_{4}$ and thermalized $\alpha \mathrm{s}$ ), and the symbol $\langle X\rangle$ indicates that $X$ is a volume-averaged quantity. Note that, for illustrative purposes and to show more clearly general trends, only a few time points have been selected from the complete time traces shown in $[1-4,15,16]$, the original data (apart from the $P_{\mathrm{NBI}}$ time trace) having also been binned over $\pm 25 \mathrm{~ms}$ for graphical purposes.

First, note that the maximum in $P_{\alpha \mathrm{FUS}}$ is obtained around $t=13.8 \mathrm{~s}$, and the peak $T_{\mathrm{e} 0}$ is obtained within one $\tau_{\alpha \mathrm{e}}$, around $t=14.05 \mathrm{~s}$. Second, note that the peak $T_{\mathrm{i} 0}$ is obtained very close to the time of the peak in $P_{\alpha \mathrm{FUS}}$, perhaps even 20 to $50 \mathrm{~ms}$ before it (however, this corresponds to the time resolution on the $T_{\mathrm{i}}$ measurements, hence it is not possible to resolve this timing relation more precisely). Third, the $T_{\mathrm{i} 0}$ rise is much larger than the $T_{\mathrm{e} 0}$ rise as a function of $P_{\alpha \mathrm{FUS}}$ : for \#42856, we have $T_{\mathrm{e} 0} \sim 8.5 \mathrm{keV}$ at $P_{\alpha \mathrm{FUS}}=0$ and $T_{\mathrm{e} 0} \sim 10.2 \mathrm{keV}$ at $P_{\alpha \mathrm{FUS}}=1 \mathrm{MW}$, hence $\Delta T_{\mathrm{e} 0} / P_{\alpha \mathrm{FUS}} \sim 1.7 \mathrm{keV} \mathrm{MW}^{-1}$, whereas $T_{\mathrm{i} 0} \sim 11.3 \mathrm{keV}$ at $P_{\alpha \mathrm{FUS}}=0$ and $T_{\mathrm{i} 0} \sim 17.2 \mathrm{keV}$ at $P_{\alpha \mathrm{FUS}}=1 \mathrm{MW}$, giving $\Delta T_{\mathrm{i} 0} / P_{\alpha \mathrm{FUS}} \sim 5.9 \mathrm{keV} \mathrm{MW}^{-1}$, i.e. $\left(\Delta T_{\mathrm{i} 0} / P_{\alpha \mathrm{FUS}}\right) /\left(\Delta T_{\mathrm{e} 0} / P_{\alpha \mathrm{FUS}}\right) \sim 3$. Fourth, the rise time $t_{\mathrm{Rx}}=\left(\mathrm{d} \log \left(T_{x 0}\right) / \mathrm{d} t\right)^{-1}$ for the ion and electron temperatures in the plasma centre are very different: whereas $\tau_{R e} \sim 1.4 \mathrm{~s}$ is broadly consistent with the time scale of the slowingdown of the $\alpha$ s on electrons $\left(\tau_{\alpha \mathrm{e}} \sim 1.2 \mathrm{~s}\right), \tau_{\mathrm{Ri}} \sim 800 \mathrm{~ms}$ corresponds to a time scale that is about five times faster than those associated with the slowing-down of the $\alpha$ s on the ions and the electron-ion energy equipartition time, $\tau_{\alpha \mathrm{i}} \sim$ $\tau_{\mathrm{ei}} \sim 4 \mathrm{~s}$ to $\tau_{\alpha \mathrm{i}} \sim \tau_{\mathrm{ei}} \sim 5 \mathrm{~s}$. Fifth, the ion temperature decays much faster than the electron temperature after the peak $P_{\alpha \mathrm{FUS}}$, on time scales that are again much faster than the energy equipartition time with the electrons, this even with a constant $P_{\mathrm{NBI}}$ (which, we remind the readers, was injected at the energy where the collisional heating of the ions is maximized). Hence, it is phenomenologically intuitive that some mechanisms other than collisional heating and ion- electron energy equipartition must be at play not only to produce this much larger than expected, but also to saturate the increase of, and then eventually reduce, the ion temperature.

Again for illustrative purposes, figures $2(a)$ and $(b)$ show, respectively, the radial profiles of the main background plasma parameters (electron density $n_{\mathrm{e}}$ and temperature $T_{\mathrm{e}}$, ion temperature $\left.T_{\mathrm{i}}\right)$ and of their scale lengths $\left(R_{0} / L_{n_{\mathrm{e}}}, R_{0} / L_{T_{\mathrm{e}}}\right.$, $R_{0} / L_{T_{\mathrm{i}}}$, with $L_{X}=-(\mathrm{d} \log (X) / \mathrm{d} r)$ and $R_{0}$ being the magnetic axis position) together with the density of fusionborn $\alpha \mathrm{s}\left(n_{\alpha}\right)$ at birth, and the fusion alpha power density $\left(\rho_{\alpha \mathrm{FUS}}\right)$. In figures $2(a)$ and $(b) \sqrt{\psi_{\mathrm{N}}}=(\psi(r) / \psi(r=$ a) $)^{1 / 2}$ is the radial coordinate, expressed in units of the poloidal flux $\psi(r)$, so that $\sqrt{\psi_{\mathrm{N}}}(r) \sim r / a$ for the case of a monotonic safety factor profile, as obtained in these discharges. Note that the shape of the electron density and temperature and ion temperature profiles do not vary significantly as a function of $n_{\alpha}$ and $\rho_{\alpha \mathrm{FUS}}$, at least up to $\sqrt{\psi_{\mathrm{N}}} \sim 0.8$, over the time evolution of the alpha-heating phase, as clearly seen when looking at the scale lengths for these quantities. This indicates that the time evolution of $T_{\mathrm{i} 0}$ versus $\mathrm{n}_{\alpha}$ and $P_{\alpha \mathrm{FUS}}$ cannot solely be associated with changes in the background plasma properties due to direct collisional heating by the $\alpha \mathrm{s}$. Hence, this suggests a role for the fusion-born $\alpha$ s that is different from the one that could be simply associated with these particles being 'tracer' elements in the plasma.

The anomalous ion heating data are finally summarized in figure 3 for the three discharges in the alpha-heating experiment that illustrate the full range of variation in the DT 

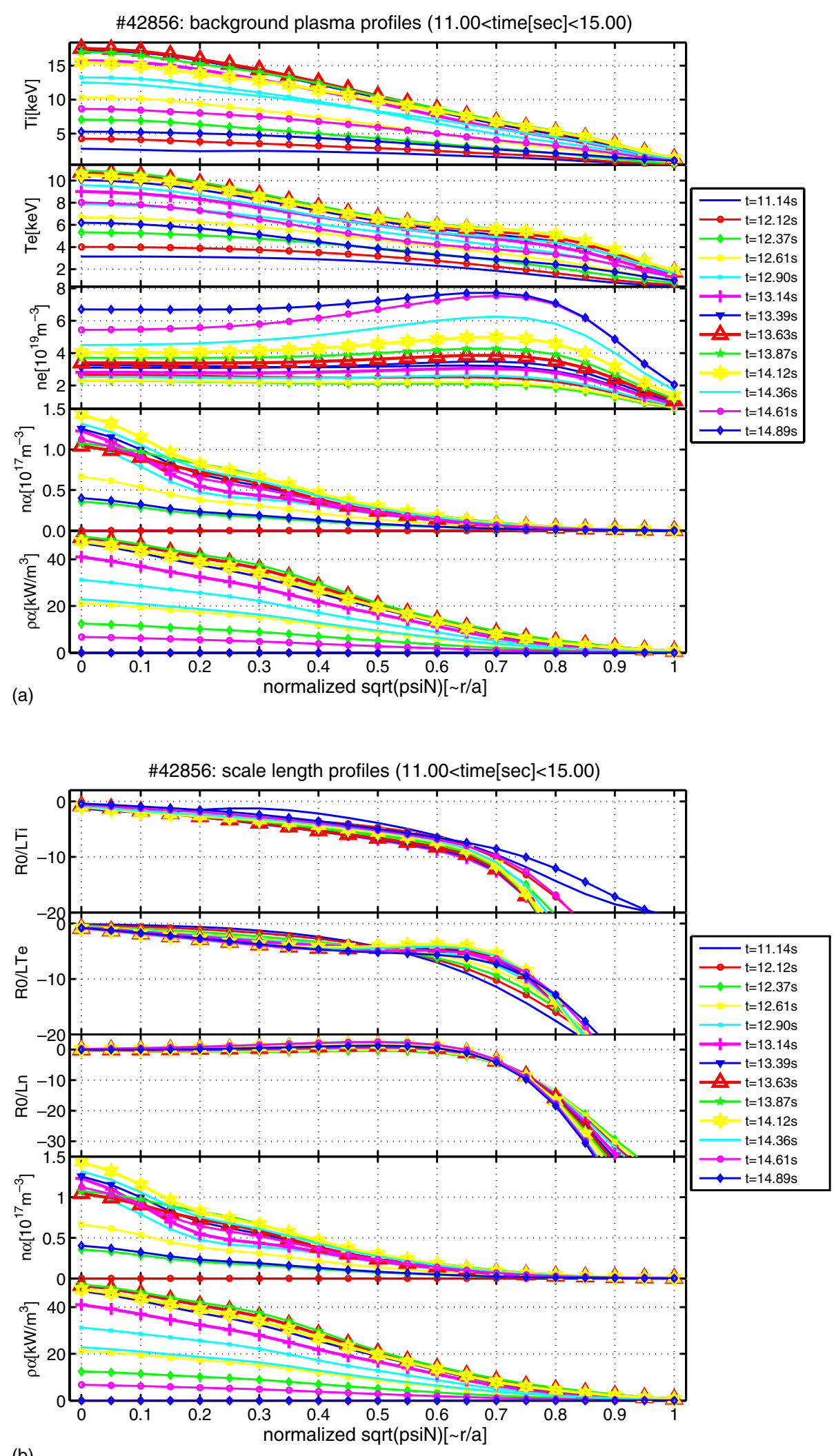

(b)

Figure 2. (a) Radial profiles of the main background plasma parameters, density at birth of the fusion-born $\alpha \mathrm{s}\left(n_{\alpha}\right)$ and fusion alpha power density $\left(\rho_{\alpha \mathrm{FUS}}\right)$ for $\# 42856$, plotted over the NBI heating phase and selecting some of the time points shown in figure 1 for clarity of presentation. (b) Radial profiles of the scale lengths for the electron density and temperature and for the ion temperature together with the density at birth of the fusion-born $\alpha \mathrm{s}\left(n_{\alpha}\right)$ and the fusion alpha power density ( $\left.\rho_{\alpha \mathrm{FUS}}\right)$, using the same format as in $(a)$. 


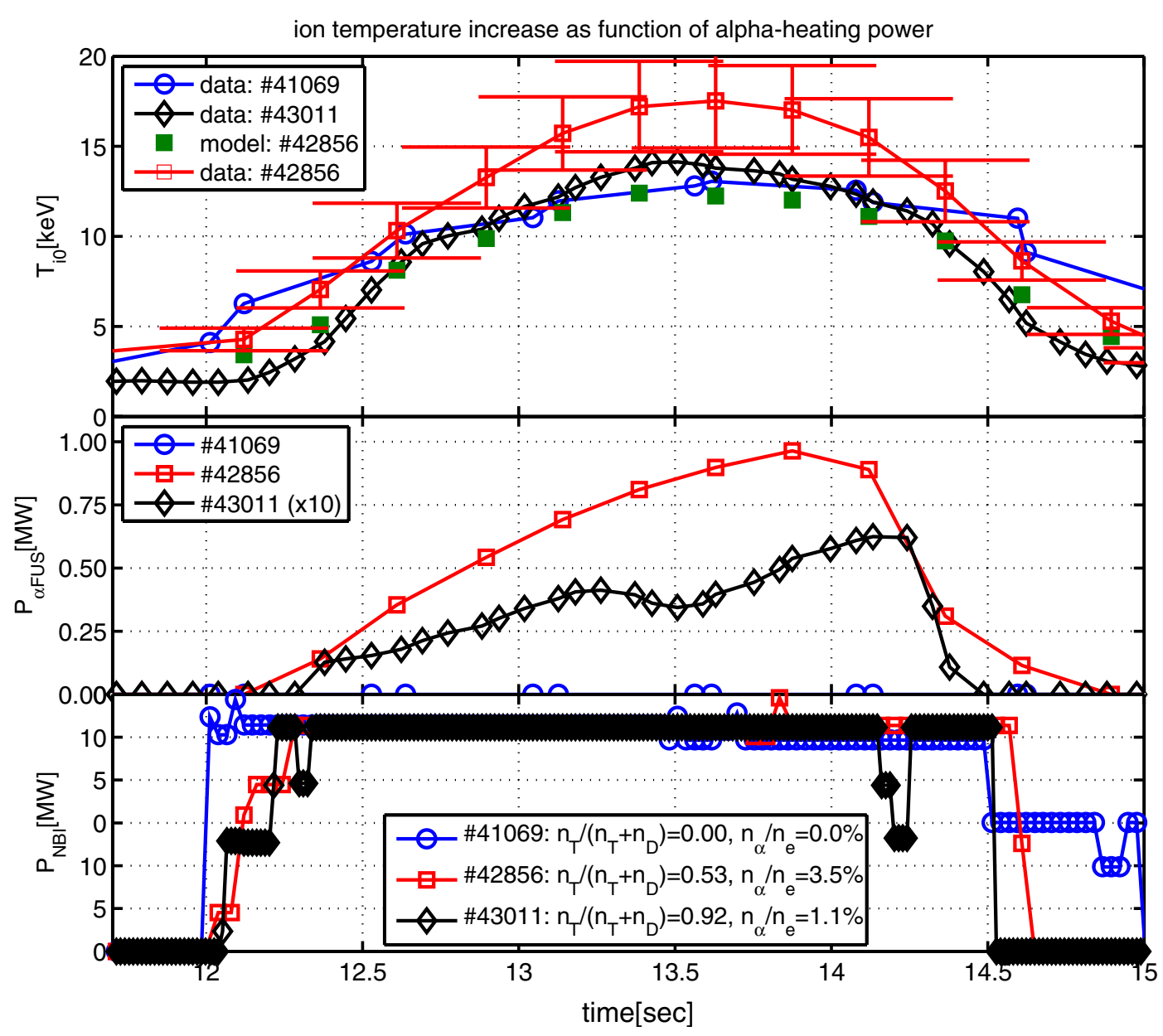

Figure 3. Summary overview of the data for the anomalous ion heating observed in the alpha-heating experiment. The ion temperature increase over the prediction is plotted as a function of the alpha-fusion power for the three discharges corresponding to the extreme in the DT fuel ratio, $P_{\alpha \text { FUs }}$ and $n_{\alpha} / n_{\mathrm{e}}$ experimental scan. Note that, for illustrative purposes and to show more clearly general trends, only a few time points have been selected from the complete time traces shown in $[1-4,15,16]$, the original data (apart from the $P_{\mathrm{NBI}}$ time trace) having also been binned over $\pm 25 \mathrm{~ms}$ for graphical purposes.

mixture ratio $n_{\mathrm{T}} /\left(n_{\mathrm{D}}+n_{\mathrm{T}}\right)$, alpha-fusion power $P_{\alpha \mathrm{FUS}}$ and $\alpha \mathrm{s}$ concentration at their birth energy $n_{\alpha} / n_{\mathrm{e}}$. Note that there is a clear $\Delta T_{\mathrm{i} 0} \approx 3 \mathrm{keV}$ excess in the core ion temperature for $n_{\mathrm{T}} /\left(n_{\mathrm{D}}+n_{\mathrm{T}}\right)=0.53$ (discharge \#42856) at the time of the maximum in $P_{\alpha \mathrm{FUS}}$ compared with the value expected using the same transport model that correctly predicted the $T_{\mathrm{e}}$ and $T_{\mathrm{i}}$ evolution (see $[1-4,11,15,16]$ for details) for $n_{\mathrm{T}} /\left(n_{\mathrm{D}}+n_{\mathrm{T}}\right)=$ 0 , even accounting for the residual direct collisional heating by the $\alpha \mathrm{s}$ on the thermal ions. Finally, note also that during the $P_{\mathrm{NBI}}$ flat-top phase $T_{\mathrm{i} 0}$ for $n_{\mathrm{T}} /\left(n_{\mathrm{D}}+n_{\mathrm{T}}\right)=0.92$ (discharge \#43011) is very similar to $T_{\mathrm{i} 0}$ for $n_{\mathrm{T}} /\left(n_{\mathrm{D}}+n_{\mathrm{T}}\right)=0$ (discharge \#41069), hence an isotopic effect can contribute to but cannot on its own explain the $T_{\mathrm{i} 0}$ excess for $n_{\mathrm{T}} /\left(n_{\mathrm{D}}+n_{\mathrm{T}}\right)=0.53$ (discharge \#42856). This set of observations has prompted us to investigate a possible direct effect of the $\alpha$ s that had not been previously considered.

\section{GENE simulations of the ion and electron drift-wave turbulence spectra}

The nonlinear interaction between micro-turbulent fields and high-energy ions is a challenging phenomenon to model.
Numerically, different time and length scales need to be resolved, such as the fast particle motion and the slow evolution of the micro-turbulent fields. Furthermore, a large number of grid points in real and velocity space are needed for each simulation and the rather large computational demand is hard to fulfil with present-day resources. Finally, a strong limitation is given by the $\Delta f$ approximation employed in the vast majority of gyro-kinetic codes. Specifically, the nonlinear evolution of the multi-dimensional distribution of fusion-born $\alpha$ s would need to be resolved, in principle, without any assumption on their stationary distribution function.

Recent upgrades of the well-known gyro-kinetic code GENE [8] can ease the qualitative analysis of the turbulent damping and excitation mechanisms. In particular, a new eigensolver was developed, allowing the characteristic frequencies and growth rates of the underlying micro-instabilities to be obtained more readily. The newly implemented numerical model guarantees optimal performance together with the accuracy of the physical model embedded in the GENE code. All simulations are performed employing the local version of GENE with the geometric properties of the equilibrium from the plasma discharge \#40365 (where neither tritium nor $\alpha$ s were present), taking 
\#40365, time=13.3sec; ITG (left) and TEM (right) turbulence spectrum
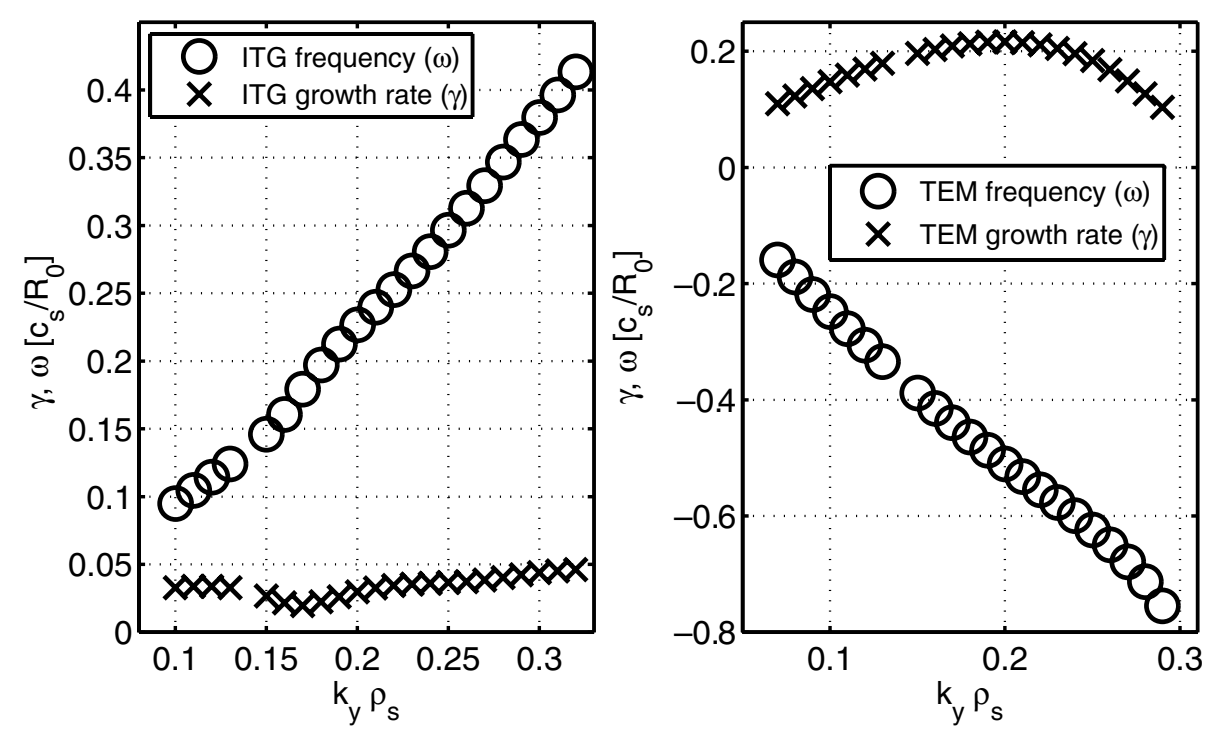

Figure 4. In the left panel, the growth rate $\gamma$ and angular frequency $\omega$ of turbulent modes rotating in the ion-drift direction (ITG modes) for \#40365 at the time-point T1 (same $T_{\mathrm{e} 0}$ ), plotted as a function of $k_{\mathrm{y}} \rho_{\mathrm{s}}$; in the right panel, the same quantities for turbulent modes rotating in the electron-drift direction (TEM, with negative frequencies). The values for the growth rate and frequency have been normalized to the local value of the ion acoustic frequency $c_{\mathrm{s}}(R=3.5 \mathrm{~m}) / R_{0}$.

the background plasma parameters at mid-radius $(R=3.5 \mathrm{~m}$, $\left.\sqrt{\psi_{\mathrm{N}}}=0.5 \sim r / a\right)$. This choice is motivated by the TRANSP calculations suggesting that a reduction in $\chi_{i}$ around midradius was needed to explain the ion temperature increase in the alpha-heating experiment [4], and by the radial localization of the turbulence eigenfunction extracted from the crosscorrelation data that will be shown later in section 4 . Finally, note that a fixed electron beta $\beta_{\mathrm{e}}=1.1 \%$, as obtained in the lowest $\beta_{\mathrm{e}}$ discharge (\#40365), is used to solely focus on the isotopic and alpha particle effects.

Four main species with different concentrations characterize the simulated JET plasmas. The main bulk ions are deuterium and tritium, whose densities are changed according to the values presented in table 1. Data from table 1 are then also used to set the density of the third species, an additional Maxwellian population of helium ions representing the fusionborn $\alpha$ s. A population of electrons is finally introduced so that quasi-neutrality is locally satisfied.

As previously remarked, fusion products behave in a nonthermal way. Their distribution, in the absence of strong anisotropies in the background plasma and in the heating schemes, as obtained using NBI in contrast to what could have been obtained using ICRF heating, is peaked at $3.5 \mathrm{MeV}$ in energy and uniformly distributed in pitch angle. A Maxwellian distribution can nevertheless be employed to represent these particles, following the procedure described by Estrada-Mila et al [27]. An equivalent temperature can then be used to set the correspondence with the energy stored by the fusion products. This approach represents a good qualitative approximation of the reality, which is more correctly represented by a slowingdown distribution function, as recently shown [28].

As the background for our turbulence simulations, we start from the ansatz that there is a direct link between electron and ion drift-wave micro-instabilities, such as trapped electron modes (TEMs) and ion and electron temperature gradient
(ITG and ETG, respectively) turbulence, and the temporal evolution of the thermal electron and ion temperatures through modifications to the electron and ion diffusivity and thermal conductivity [29]. The nonlinear evolution of small scale turbulence, such as TEM, ETG and ITG, consistently reflects the linear behaviour of the micro-instabilities [30-32]. Hence, as larger linear growth rates $(\gamma)$ always induce stronger nonlinear heat fluxes, and with the currently employed local version of the GENE code we are only able to study the linear features of the background micro-instabilities, we use the numerically obtained linear growth rate as a more readily available proxy to evaluate the strength of the saturated turbulence, i.e. readily linking the calculated $\gamma$ from GENE to the turbulence amplitude measurements that will be shown in section 4.

A first set of simulations is needed to identify the key features of the turbulence characterizing the baseline plasma scenario. We thus perform a scan in the perpendicular wavenumber $k_{y}$ for \#40365. The values for the temperature and density scale lengths for the background plasma species (deuterium and electrons) are chosen from the experimental dataset at the time-point T1. The local electron beta is set to $\beta_{\mathrm{e}}=1.1 \%$, a value sufficiently far from the threshold of kinetic ballooning modes, as found in preliminary scans that were performed previous to this analysis. We therefore expect ITG modes and TEMs to be the predominant micro-instabilities present in this plasma. Our expectations are confirmed by the results of the numerical simulations, illustrated in figure 4, where the growth rate $\gamma$ and the angular frequency $\omega$ of turbulent modes rotating in the ion (ITG modes) and electron (TEMs) drift directions are plotted as a function of $k_{y} \rho_{\mathrm{s}}$, where $\rho_{\mathrm{s}} \sim \sqrt{T_{\mathrm{e}}} \sqrt{m_{\mathrm{D}}}$ is the Larmor radius of the background deuterons computed with the electron temperature. The co-existence of ITG mode and TEMs over the simulated domain is clearly visible, with the largest instability in the 

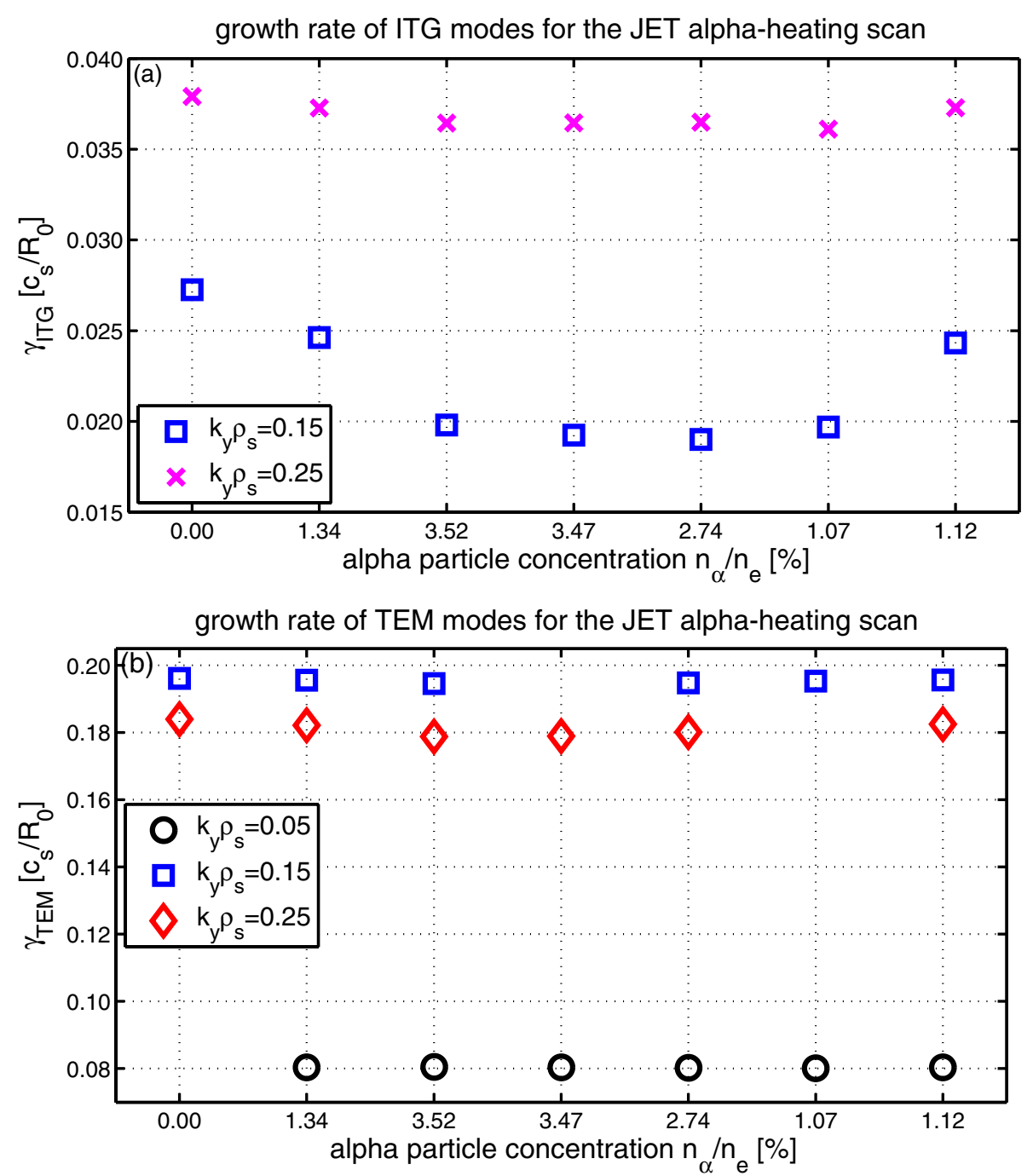

Figure 5. $(a),(b)$ Growth rate of ITG (top frame, $(a)$ ) and TEMs (bottom frame, $(b)$ ), plotted as a function of the alpha particle concentration $n_{\alpha} / n_{\mathrm{e}}$ for seven of the nine discharges constituting the JET alpha-heating scan (see table 1 for their ordering: \#41069 and $\# 43491$ were not used for this analysis as they both had $n_{\alpha} / n_{\mathrm{e}}=0$ as in \#40365, which is the data point with $n_{\alpha} / n_{\mathrm{e}}=0$ shown here). The ITG and TEM growth rates are plotted separately for different values of $k_{y} \rho_{\mathrm{s}}$, and considering only the contribution of fully thermalized $\alpha$ s with $T_{\alpha}=80 T_{\mathrm{e}}$. As in figure 4, the growth rate $\gamma$ is normalized with respect to the local value of the ion acoustic frequency $c_{\mathrm{s}}(R=3.5 \mathrm{~m}) / R_{0}$, and it is computed taking the background plasma parameters for \#40365 at the time-point T1.

ion-drift direction peaking in the toroidal mode number range $65<n<120$. The ITG frequency of this mode sits between 20 and $40 \mathrm{kHz}$ in the plasma rest frame. Then, in the absence of tritium and $\alpha \mathrm{s}$, both micro-instabilities in the ion and electrondrift direction are present.

Damping of ITG (and TEM) turbulence could be prompted by a combination of the plasma isotopic mixture and direct stabilization from the $\alpha \mathrm{s}$. To study these hypotheses, a second set of simulations is required so as to compute the growth rate of ITG modes $\left(\gamma_{\mathrm{ITG}}\right)$ and TEMs ( $\gamma_{\text {TEM }}$ ) for different wavenumbers $k_{y} \rho_{\mathrm{s}}$ as a function of the alpha particles' concentration $\left(n_{\alpha} / n_{\mathrm{e}}\right)$ and their equivalent Maxwellian temperature $\left(T_{\alpha}\right)$. Figure 5 shows the value of $\gamma_{\text {ITG }}$ (top frame, figure $5(a)$ ) and $\gamma_{\text {TEM }}$ (bottom frame, figure $5(b)$ ) as calculated by GENE, using for consistency the background plasma parameters for $\# 40365$ at the timepoint T1. Note also that for these simulations we have only considered the contribution of fully thermalized $\alpha$ s with
$T_{\alpha}=80 T_{\mathrm{e}}$. As shown in figure $5(a)$, the maximum value of $\gamma_{\text {ITG }}$ is obtained for $k_{y} \rho_{\mathrm{s}}=0.25$. A clear reduction in the growth rate of ion-drift wave modes, particularly for the case $k_{y} \rho_{\mathrm{s}}=0.15$, corresponding to the stabilization of ITG turbulence, is observed when tritium and deuterium have similar densities (i.e. a 50-50 DT fuel mixture), giving the largest $n_{\alpha} / n_{\mathrm{e}} \sim 3.5 \%$ alpha particle concentration. Despite some missing points due to limitations in the GENE algorithm (as reported in [33]), the stabilization of ITG modes in the lower $k_{y} \rho_{\mathrm{s}}$ region (the one driving the strongest ion transport) is clear. Regarding the modes rotating in the electron-drift direction, we note in figure $5(b)$ that the stabilization previously observed for ITG modes with a 50-50 DT fuel mixture is not present for TEM, and an increase in their growth rate is actually apparent for the largest $k_{y} \rho_{\mathrm{s}}$ modes. TEMs are found to respond rather differently to the presence of $\alpha$ s and to the varying DT mixture. In fact, no change in the TEM evolution is expected when fusion products are present. Therefore, any increase in the 

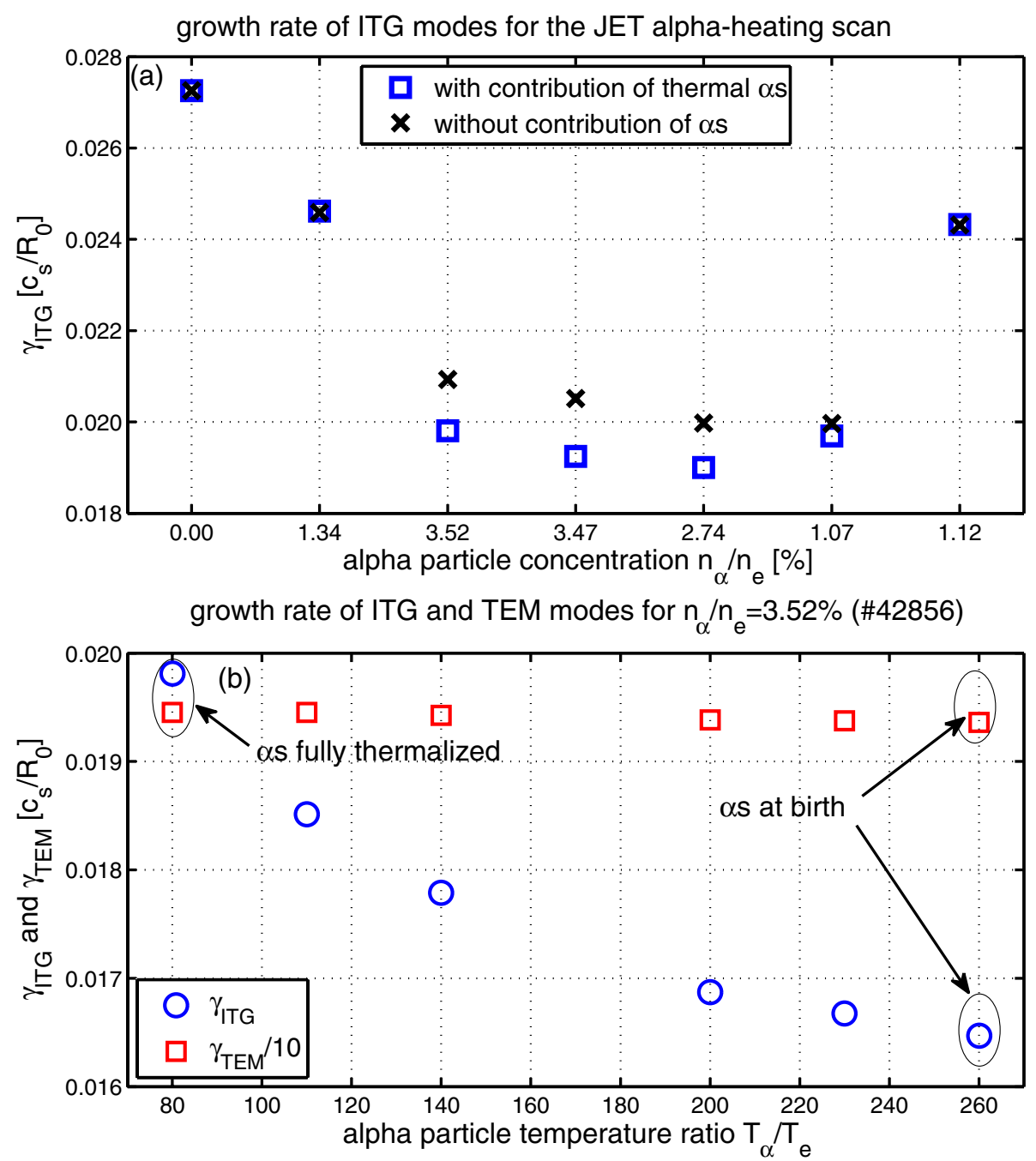

Figure 6. Top frame, (a) growth rate of ITG modes calculated by GENE for $k_{y} \rho_{\mathrm{s}}=0.15$ with and without thermal $\alpha$ s for the seven $n_{\alpha} / n_{\mathrm{e}}$ cases analysed in figure 5, again taking the background plasma parameters for \#40365 at T1 for consistency with figures 4, 5. Bottom frame, (b) growth rate of ITG modes and TEMs for discharge \#42856 with $n_{\alpha} / n_{\mathrm{e}}=3.52 \%$ as a function of $T_{\alpha}$, computed using the corresponding background plasma parameters at the time-point T1, from $T_{\alpha}=80 T_{\mathrm{e}}$ (fully thermalized $\alpha \mathrm{s}$ ), to $T_{\alpha}=260 T_{\mathrm{e}}(\alpha \mathrm{s}$ at birth). As in figure 4 , the growth rate is normalized with respect to the local value of the ion acoustic frequency $c_{\mathrm{s}}(R=3.5 \mathrm{~m}) / R_{0}$.

electron temperature as $\alpha$ s are created is indeed entirely due to classical fusion product thermalization $[2,3]$.

As both plasma mixture and alpha particle effects are contained in the picture emerging from figure 5 , a third step is required to clarify (at least qualitatively) the relative importance of these two terms. Figure 6(a) shows $\gamma_{\mathrm{ITG}}$ for fixed $k_{y} \rho_{\mathrm{s}}=0.15$ as a function of $n_{\alpha} / n_{\mathrm{e}}$, this time with and without the fully thermalized $\alpha$ s, again taking the background plasma parameters for \#40365 at the time-point T1 for consistency. We clearly see that $\alpha$ s have little to no effect at low concentrations, as $\gamma_{\text {ITG }}$ remains practically constant for values of $n_{\alpha} / n_{\mathrm{e}}<2 \%$. Increasing the density of fusion products, i.e. for cases with $n_{\alpha} / n_{\mathrm{e}}>2.5 \%$, causes a reduction in $\gamma_{\mathrm{ITG}}$, hence in $\chi_{\mathrm{i}} \propto \Sigma \gamma_{\mathrm{ITG}} / k_{y}^{2}$ (which can be estimated by summing $\gamma_{\text {ITG }} / k_{y}^{2}$ over the spectrum of ITG modes [34]), when the $\alpha$ s are included. We have then increased the equivalent Maxwellian temperature of the $\alpha$ s population for the specific case $n_{\alpha} / n_{\mathrm{e}}=3.52 \%$ (discharge \#42856, using the actual background plasma parameters for this discharge at the timepoint T1) from $T_{\alpha}=80 T_{\mathrm{e}}$, corresponding to fully thermalized $\alpha \mathrm{s}$ (which is the situation expected at the time points T2 of the maximum in $T_{\mathrm{e} 0}$, see table 1 ), to $T_{\alpha}=260 T_{\mathrm{e}}$, corresponding to $\alpha$ s at their birth energy. This second case simulates the role of $\alpha$ s that have not yet had the time to fully thermalize, which is the situation expected at the time points $\mathrm{T} 1$ of the same $T_{\mathrm{e} 0}$ (as given in table 1). This allows assessing the possible beneficial effect for ITG (and TEM) stabilization introduced by a larger energy of the $\alpha$ s. Given the large energy of the $\alpha$ s at birth, an important influence of the $\alpha$ s on the stabilization of ITG modes is expected. As shown in figure $6(b)$, we do indeed find that the most stable point for ITG modes corresponds to $T_{\alpha}=260 T_{\mathrm{e}}$, indicating that $\alpha$ s close to their birth energy contribute most to the stabilization of ITG turbulence. Similarly to the results shown in figure $5(b)$, we find that the TEM growth rate is essentially unaffected by the increase in $T_{\alpha} / T_{\mathrm{e}}$.

Summarizing the GENE results for this specific case with $n_{\alpha} / n_{\mathrm{e}}=3.52 \%$, we find that the linearly calculated ITG growth rate is $\gamma_{\mathrm{ITG}}=0.0209$ without $\alpha \mathrm{s}, \gamma_{\mathrm{ITG}}=0.0198$ with $\alpha$ s at $T_{\alpha} / T_{\mathrm{e}}=80$, and finally $\gamma_{\mathrm{ITG}}=0.0165$ with $\alpha \mathrm{s}$ at $T_{\alpha} / T_{\mathrm{e}}=260$, giving an overall reduction of about $20 \%$ 
in the linear estimate of $\chi_{\mathrm{i}} \propto \gamma_{\text {ITG }}$ for the single wavenumber $k_{y} \rho_{\mathrm{s}}=0.15$. Note also that it is well known that a larger $\beta_{\mathrm{e}}$ can contribute to mitigate the strength of ITG micro-instabilities in high temperature discharges [35], i.e. specifically those with more $\alpha$ s. The simulations presented here assume the same and lowest value of $\beta_{\mathrm{e}}=1.1 \%$ for all the discharges in the alphaheating scan to solely focus on the effect of the $\alpha \mathrm{s}$, hence this further $\beta_{\mathrm{e}}$ stabilization effect is not taken into account here.

The $T_{\alpha}$ dependence shown in figure $6(b)$ indicates that high-energy D-NBI and T-NBI ions cannot be responsible for the ITG stabilization mechanism, as the beam injection energy $(\sim 150 \mathrm{keV})$ is only about $10 \times T_{\mathrm{e}}$, whereas at least $T_{\alpha} \sim 80 \times T_{\mathrm{e}}$ is needed to observe any effect in the GENE simulations. Moreover, ICRF heating was used in a series of comparison discharges to replace the fusion-born, velocity-space isotropic $\alpha$ s with still multi-MeV-energy, but anisotropic, fast hydrogen and deuterium ions. In those cases, no anomalous level of ion heating was observed. Finally, a mechanism for ITG turbulence suppression similar to the alpha-channelling scheme [36] can be discounted since not only no sign of the required bump-on-tail distribution function for the $\alpha$ s was observed, but also such a scheme would have required about $35 \%$ of the alpha-energy to be taken from the electron channel for use by the alpha-channelling turbulence suppression mechanism, which is not compatible with the observed increase in $T_{\mathrm{e}}$ being due to in excess of $85 \%$ of the alpha-energy being collisionally transferred to the electrons.

\section{Measurements of the ion and electron drift-wave turbulence spectra}

The measurement of the turbulence spectra for the JET alpha-heating experiment was performed using magnetic data, obtained via Mirnov-type pick-up coils mounted on the lowand high-field side vessel walls, ECE and O- and X-mode reflectometry. These fluctuation data were acquired using the central acquisition and triggering system (the so-called CATS diagnostic [37]) and the KC1F diagnostic system [38]. These two diagnostic systems served different purposes: whereas the CATS system provided concurrent and synchronized acquisition of a very large number of channels ( $>200$, including magnetics, ECE and reflectometry measurements) for short time windows (up to 7, the duration of each one not exceeding $150 \mathrm{~ms}$ ) and at moderate frequency (up to $250 \mathrm{kHz}$ ), the $\mathrm{KC} 1 \mathrm{~F}$ systems had eight channels (mostly magnetics), which were, however, acquired continuously at $1 \mathrm{MHz}$ over four seconds in all but two (\#40365 and \#41069) of the discharges analysed here (where the data acquisition time window lasted only one second due to RAM limitations).

For the purpose of the analysis reported here, the CATS and $\mathrm{KC} 1 \mathrm{~F}$ data have been synchronized (a known time lag of approximately $17 \mu$ s existed in 1997 between the two diagnostic systems) and combined together whenever possible. As a full end-to-end frequency-dependent calibration exists for the magnetics channels acquired through the $\mathrm{KC} 1 \mathrm{~F}$ system [38], whereas such calibration is not available for the same pick-up coils when acquired through the CATS system, the latter have been rescaled to match the FFT amplitude of the $\mathrm{KC} 1 \mathrm{~F}$ data. However, a detection problem exists for the internal fluctuation measurements in that not enough of these data were acquired via the $\mathrm{KC} 1 \mathrm{~F}$ diagnostic. As no frequency-dependent end-to-end calibration is available for the CATS system, the accuracy in the reconstruction of the radial structure of the turbulence spectrum is therefore significantly reduced. Moreover, the reflectometry and the ECE fluctuation measurements were not consistently set up between all the discharges in the alpha-heating experiment, and the signal-tonoise ratio $(S / N)$ was also very poor for the reflectometry data acquired via the CATS diagnostic system, thus further reducing the accuracy of these internal fluctuation measurements.

Regarding the magnetic data acquired through the $\mathrm{KC1F}$ diagnostic system and their error analysis, we first note that the measured magnetic field signal $\left|\delta B_{\text {MEAS }}\right|$ corresponding to the one-count level is a frequency-dependent quantity related to the number of digitizer bits $(=12)$, the fullscale voltage measurement ( $=10 \mathrm{~V}$-peak), the effective area $\left(\mathrm{NA}_{\mathrm{EFF}}=0.064 \mathrm{~m}^{2}\right)$ and the end-to-end transfer function for each individual pick-up coil. This gives a value for the minimum measurable magnetic field $\left|\delta B_{\text {MEAS }}\right|$ producing at least a one-count signal in the $\mathrm{KC} 1 \mathrm{~F}$ digitizers around $0.5 \mathrm{mG}$ at $10 \mathrm{kHz}$ and $0.01 \mathrm{mG}$ at $200 \mathrm{kHz}$. For our error analysis, we use the Poisson statistics to estimate the relative error on the $\mathrm{KC} 1 \mathrm{~F}$ digitizer count, and the estimated error on the end-to-end transfer function [38]. Accordingly, an error thresholding scheme (via the $\lambda_{\text {NORM }}$ parameter, see the appendix) is set in the SRS calculations for the measured magnetic signal $\left|\delta B_{\text {MEAS }}(\omega)\right|$ in the drift-wave frequency range, from a minimum value which corresponds to three counts at $10 \mathrm{kHz}$. We then use the convergence criterion on the SRS algorithm to determine a further thresholding scheme for the individual toroidal components $\left|\delta B_{\text {MEAS }}(\omega, n)\right|$, which is implemented via an ad hoc cut-off on the amplitude of the calculated modes [7,39] (see the appendix and references therein for more details on this point). Finally, for the SRS calculations we use an atom base extending up to $|n|=500$, and we consider the SRS solutions to be corresponding to physical modes (i.e. not numerical artefacts) up to $|n| \sim 100$ (see again [7,39], the appendix and references therein for more details on this point). This value of the maximum detectable toroidal mode number is around three times the highest toroidal mode number that could have been detected using the two closest pick-up coils via a linear phase fitting method [38, 40], and it is consistent with the results of dedicated simulations previously performed to assess the SRS accuracy (see [39, 41] for more details on this point).

As shown in table 1, two different time points are selected for the turbulence analysis. The first time-point (T1) is taken at the beginning of the $P_{\mathrm{NBI}}$ flat-top phase. At this time-point approximately the same values (within the error bars on the measurements) for the core $n_{\mathrm{e} 0}$ and volumeaveraged electron density $\left\langle n_{\mathrm{e}}\right\rangle$ and its scale length $\left\langle R_{0} / L_{n_{\mathrm{e}}}\right\rangle$, core $T_{\mathrm{e} 0}$ and volume-averaged electron temperature $\left\langle T_{\mathrm{e}}\right\rangle$ and its scale length $\left\langle R_{0} / L_{T_{\mathrm{e}}}\right\rangle$, core $T_{\mathrm{i} 0}$ and volume-averaged ion temperature $\left\langle T_{\mathrm{i}}\right\rangle$ and its scale length $\left\langle R_{0} / L_{T_{\mathrm{i}}}\right\rangle$ were obtained for all the discharges in the database. This indicates that differences in the background plasma should not affect too much the dynamics of the ITG and TEM turbulence, which in turn facilitates identifying the role of the $\alpha$ s in determining the characteristics of the turbulent spectrum in this phase of the discharge. At T1 we then have $n_{\mathrm{e} 0} \sim(2.8 \pm 0.5) \times 10^{19} \mathrm{~m}^{-3}$, 
$\left\langle n_{\mathrm{e}}\right\rangle \sim(1.7 \pm 0.4) \times 10^{19} \mathrm{~m}^{-3}$ and $\left\langle R_{0} / L_{n_{\mathrm{e}}}\right\rangle \sim-(1.0 \pm 0.3)$, $T_{\mathrm{e} 0} \sim(8.5 \pm 1.2) \mathrm{keV},\left\langle T_{\mathrm{e}}\right\rangle(5.2 \pm 0.5) \mathrm{keV}$ and $\left\langle R_{0} / L_{T_{\mathrm{e}}}\right\rangle \sim$ $-(3.5 \pm 0.7), T_{\mathrm{i} 0} \sim(12.0 \pm 1.8) \mathrm{keV},\left\langle T_{\mathrm{i}}\right\rangle \sim(6.5 \pm 0.8) \mathrm{keV}$ and $\left\langle R_{0} / L_{T_{\mathrm{i}}}\right\rangle \sim-(4.5 \pm 1.0)$, where the ' \pm ' indicates the data scatter across the different discharges in the alpha-heating scan, which is to be compared with a typical $\sim 15 \%$ accuracy in the temperature and density measurements. The second timepoint (T2) is taken at the maximum in $T_{\mathrm{e} 0}$, hence corresponding to different values for the various scale lengths $\left(R_{0} / L_{n_{\mathrm{e}}}\right.$, $R_{0} / L_{T_{\mathrm{e}}}, R_{0} / L_{T_{\mathrm{i}}}$ ) that determine the stability properties of ion and electron-drift turbulence.

The first element of the analysis of the turbulence measurements deals with finding the location of the ITG and TEM instabilities. The GENE simulations presented in section 3 have shown that ITG modes and TEMs have toroidal mode numbers in the range $|n|>20$ (positive for ITG, negative for TEM), and are expected to have a similar frequency in the plasma rest frame, in the drift-wave range $20<f(\mathrm{kHz})<40$. Thus, we analyse the ECE and reflectometry data in these $n$-number (using spectral decomposition via the SRS method) and frequency range (using temporal FFT) and we find the ITG and TEM eigenfunctions through cross-correlation with the magnetic measurements. The drift-wave frequency range is defined as $\omega_{\mathrm{cs}}(r)=2 \pi\left(R_{0}+r\right) / c_{\mathrm{s}}(r)$, where $c_{\mathrm{s}}(r)=$ $\sqrt{T_{\mathrm{e}}}(r) / m_{\mathrm{p}} A_{\mathrm{EFF}}(r)$ is the local ion sound speed computed with the effective plasma mass $A_{\mathrm{EFF}}(r)=\Sigma_{\mathrm{i}} n_{\mathrm{i}}(r) m_{\mathrm{i}} / m_{\mathrm{p}} / \Sigma_{\mathrm{i}} n_{\mathrm{i}}(r)$, $m_{\mathrm{p}}$ being the proton mass, and the sum is intended over all ion species. For practical purposes we take the drift-wave frequency range to extend up to the bottom of the toroidal Alfvén eigenmode (TAE) gap. Hence, considering the radial profile of the electron temperature and Alfvén speed, and the toroidal plasma flow, the turbulence analysis is performed by summing up all the FFT frequency components from a few $\mathrm{kHz}$ up to the $\sim(100 \pm 30) \mathrm{kHz}$ range for the different discharges in the scan.

Despite the somewhat poor $S / N$ ratio, particularly for the reflectometry data, and inconsistent diagnostic settings throughout the alpha-heating experiment, that prevent obtaining a very detailed analysis, it is possible to extract in certain cases some useful information on the ITG and TEM eigenfunctions from the measurement of the reflectometry (electron density fluctuation spectra $\left.\delta n_{\mathrm{e}}(r)\right)$ and ECE electron temperature fluctuation spectra $\delta T_{\mathrm{e}}(r)$. One such case where the turbulent eigenfunction can be obtained is shown in figure 7 for \#42856 at the time-point T2 using the ECE fluctuation data (note that \#42856 had the highest value of $P_{\alpha \text { FUs }}$ and $n_{\alpha} / n_{\mathrm{e}}$ ). For \#42856, the main plasma parameters at the time-point T2 are $n_{\mathrm{T}} /\left(n_{\mathrm{D}}+n_{\mathrm{T}}\right)=0.53, n_{\alpha} / n_{\mathrm{e}}=0.032$, $T_{\mathrm{e} 0}=9.6 \mathrm{keV}, T_{\mathrm{i} 0}=15.5 \mathrm{keV}, P_{\alpha \mathrm{FUS}}=0.96 \mathrm{MW}$. As in all the turbulence measurements reported in this work, the crosscorrelation spectra have been computed removing, from all the input signals, all components associated with low- $|n|<10$ coherent modes (such as neoclassical tearing modes (NTMs) and the pre-cursors and post-cursors for sawteeth and edge localized modes (ELMs)), faulty digitizer channels (appearing as lines at constant frequency in the spectrograms shown in the top frames of figures $8-10$, and corresponding to some of the high- $|n|>50$ peaks in the bottom frames of those figures), and the saddle coil signal (essentially a mixture of the low$|n|=0-2$ components).
The largest turbulent component is found around $\sim 45 \mathrm{kHz}$ in both the ion (figure 7(a)) and electron (figure 7(b)) channels, i.e. in the drift-wave range of frequencies. This value is slightly above the drift-wave range (20 to $40 \mathrm{kHz}$ ) for a zero-flow plasma as calculated by GENE, and this upshift is consistent with the measured toroidal plasma flow being between $\sim 5 \mathrm{kHz}$ at the plasma edge and $\sim 25 \mathrm{kHz}$ in the plasma centre. The ITG and TEM eigenfunctions are both localized around $R\left(=R_{0}+r\right) \sim 3.5 \mathrm{~m} \pm 15 \mathrm{~cm}$ (top frame in figure 7), and both have an even parity (middle frame in figure 7 : there is no change of sign in the phase of the eigenfunction), i.e. corresponding to kink-type turbulent modes that do not cause the onset of a magnetic island (hence are not expected to cause radial transport of the $\alpha$ s through orbit stochasticization). Most importantly, the measured eigenfunction broadly overlaps with the plasma volume where TRANSP calculates that a reduction in the ion thermal conductivity $\chi_{\mathrm{i}}$ of a factor $\sim 2$ is needed to explain the $T_{\mathrm{i}}$ increase [4].

The equivalent magnetic turbulence strength $\delta B_{\mathrm{ECE}}(\omega, r)$ in figure 7 has been evaluated from the measured $\delta T_{\mathrm{e}}(\omega, r)$ fluctuation signal using the formalism presented in [42-44] for the optically thick plasmas of JET. Hence $\delta B_{\mathrm{ECE}}(\omega, r)$ can be expressed as

$$
\delta B_{\mathrm{ECE}}(\omega, r) \simeq\left[B_{\phi}\left(R_{0}\right) \frac{\left|\Omega_{\mathrm{e}}(r)\right|}{c \nabla T_{\mathrm{e}}(r)} f\left(\xi_{r}\right)\right] \delta T_{\mathrm{e}}(\omega, r),
$$

where $B_{\phi}\left(R_{0}\right)$ is the toroidal magnetic field at the position of the magnetic axis $R_{0}, \Omega_{\mathrm{e}}(r)$ is the radial profile of the electron cyclotron frequency, $\nabla T_{\mathrm{e}}(r)$ is the gradient in the electron temperature profile, and $f\left(\xi_{r}\right)$ represents the weighting of the ECE emission spectra $I(\omega)=\omega^{2} T_{\mathrm{e}}(r) /\left(8 \pi^{3} c^{2}\right)(1-$ $\exp (-\tau(r)))$ over the eigenfunction of the turbulence, where the optical length $\tau(r) \gg 1$ is practically constant across the plasma radial profile for the discharges considered here.

Focusing now on the turbulence spectra in the iondrift wave channel at the time-point T2, when the $\alpha$ s have fully thermalized, the ITG eigenfunction has peak amplitude $\max \left(\left|\delta B_{\mathrm{ECE}}\right|\right) \approx 20 \mathrm{mG}$ in the plasma core. Conversely, and for all the discharges in the alpha-heating scan, no such eigenfunction for the ITG turbulence can be measured at the time-point $\mathrm{T} 1$, when the $\alpha \mathrm{s}$ have not yet thermalized. This is due to the $S / N$ ratio for $\delta T_{\mathrm{e}}(\omega, r)$ being very small, giving an equivalent $\max \left(\left|\delta B_{\mathrm{ECE}}\right|\right)<2 \mathrm{mG}$ that is well below the sensitivity of the ECE fluctuation diagnostics, for which the minimum measureable $\left|\delta B_{\mathrm{ECE}}\right| \sim 5 \mathrm{mG}$. This indicates that ion-drift-wave turbulence has been reduced to below measureable levels in the plasma core when there is a sufficiently large population of fusion-born $\alpha$ s close to their birth energy. We also find that the time evolution of the ECE fluctuation signal $\delta T_{\mathrm{e}}(\omega, r, t)$ correlates very well with the time evolution of the $\delta B_{\text {MEAS }}(\omega, t)$ signal measured with pick-up coils mounted on the vessel walls. Finally, there are sufficient similarities in the frequency and toroidal mode number range and amplitude evolution between the GENE turbulence simulations and the actual turbulence measurements to suggest that the more routinely available $\delta B_{\text {MEAS }}(\omega, n)$ can be used as a proxy to evaluate drift-wave turbulence in the absence of reliable core fluctuation measurements over the whole experimental scan. 
\#42856, time=14.10sec: Eigenfunction for turbulence in the ion- drift direction

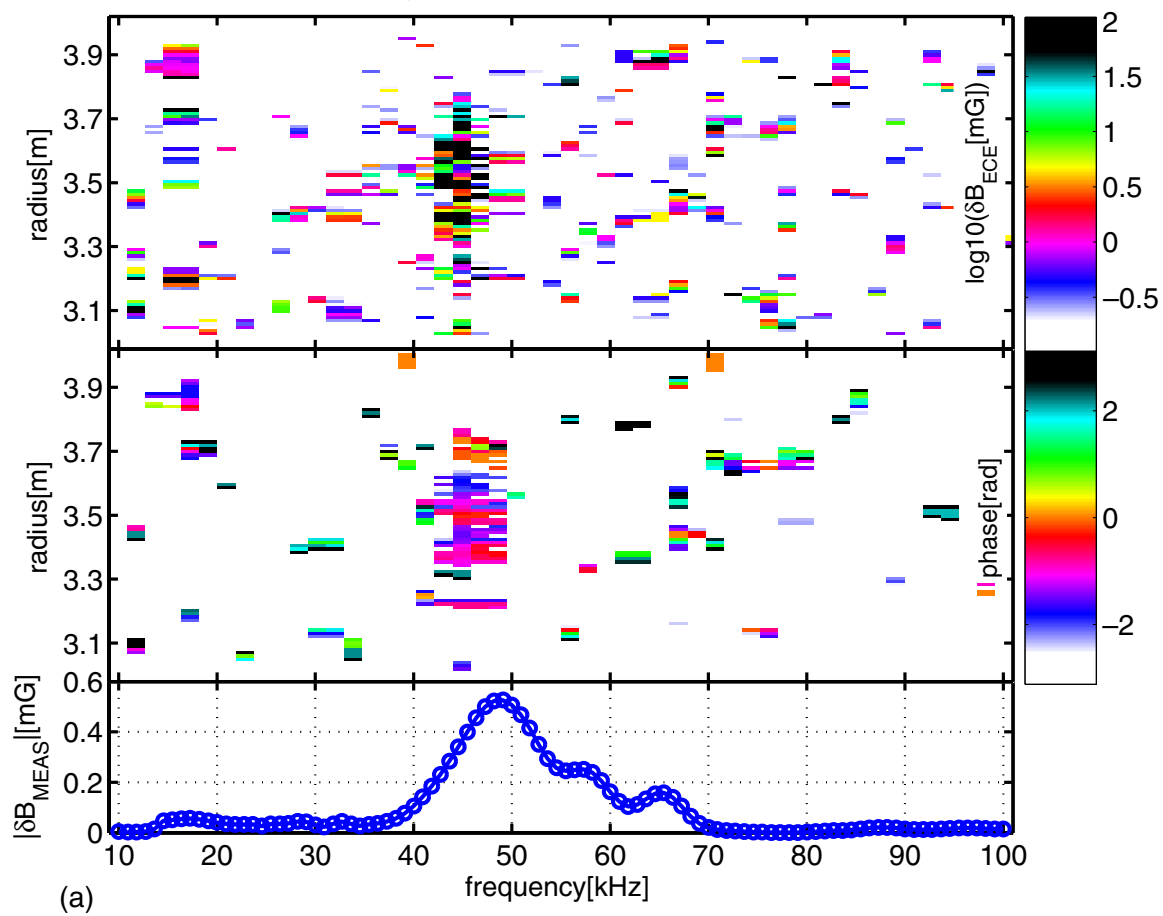

(a)

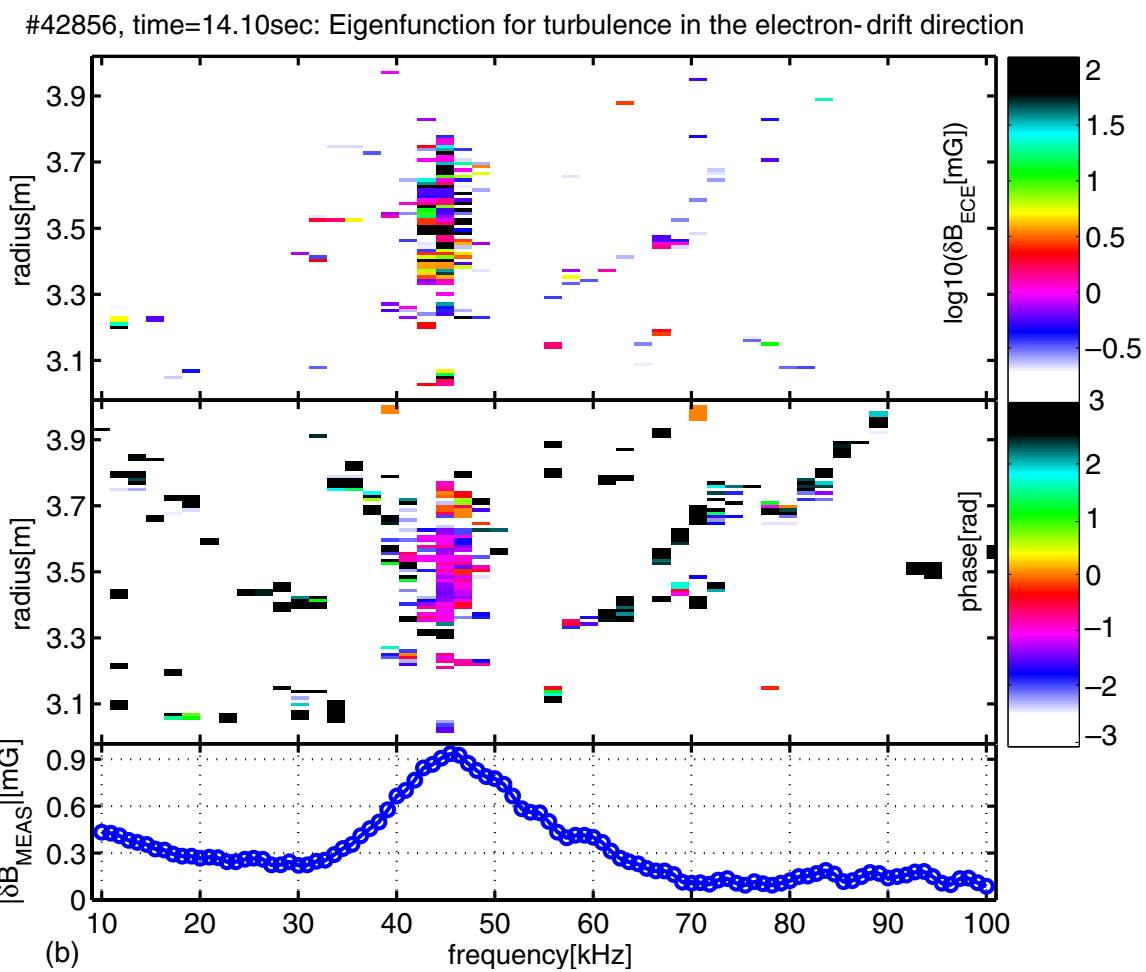

Figure 7. (a) Cross-correlation spectrum of the ITG (i.e. in the ion-drift direction, $n>20$ ) turbulence data for \#42856 at the time-point $\mathrm{T} 2=14.10 \mathrm{~s}$ (corresponding to the maximum in $T_{\mathrm{e} 0}$ ), showing a radial eigenfunction localized around $3.35<R(\mathrm{~m})<3.65$ with even parity (kink-type turbulence) for the peak frequency component observed in the drift-wave range at around $45 \mathrm{kHz} \pm 5 \mathrm{kHz}$. (b) Cross-correlation spectrum of the TEM (i.e. in the electron-drift direction, $n<-20$ ) turbulence data for \#42856 at the time-point T2=14.10 s (corresponding to the maximum in $T_{\mathrm{e} 0}$ ). The TEM eigenfunction has a similar radial structure to the ITG one (see $(a)$ ), and a slightly broader frequency spectrum, with higher amplitudes at the lower frequencies in the spectrum.

It is now very useful to discuss the important ansatz of this work of using $\delta B_{\text {MEAS }}(\omega, n)$ as a proxy for core electrostatic fluctuation measurements. First, it is paramount to remark that for the plasma discharges analysed here, where the electronic beta is rather large and can exceed $2 \%$, there is an important contribution of magnetic effects to the background turbulence (see, for instance, the introductory linear analyses of [45] and the nonlinear investigations of $[35,46,47])$. High-beta 

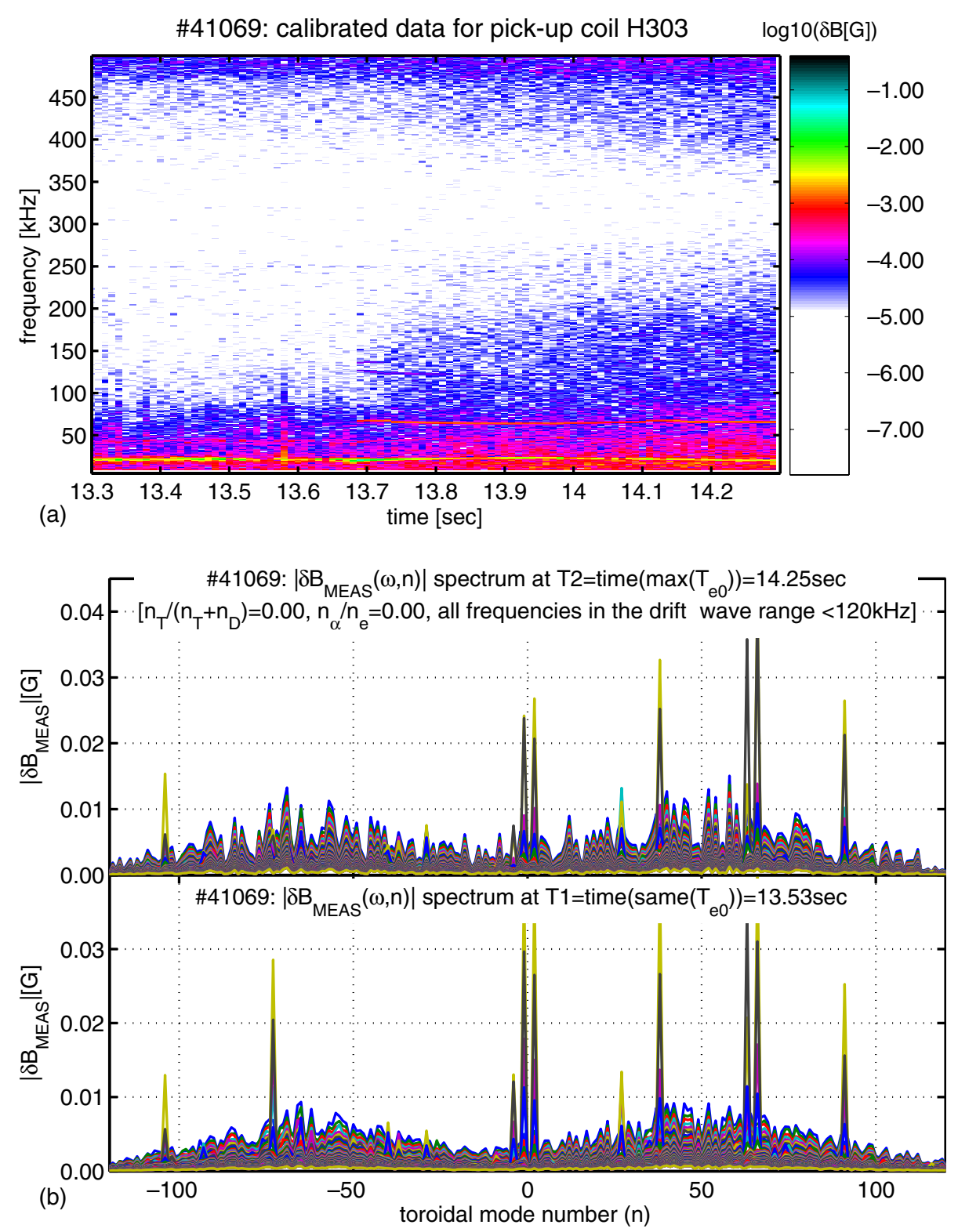

Figure 8. Measured magnetic fluctuation data for \#41069. Top frame: time/frequency spectrogram; bottom frames: auto-power spectrum at the time points $\mathrm{T} 1\left(\equiv \operatorname{same}\left(T_{\mathrm{e} 0}\right)\right)$ and $\mathrm{T} 2\left(\equiv \max \left(T_{\mathrm{e} 0}\right)\right)$.

scenarios are characterized by a marked magnetic activity and a consequent increase in the so-called 'electromagnetic' component of turbulent transport. Perturbations in $A_{\|}$( or $B_{\perp}$ ) are therefore important in the plasma core of these scenarios and can propagate towards the plasma boundary, as shown in [48], for instance. Detailed computational analyses in this area require extensive numerical simulations with a global gyro-kinetic code, which are beyond the scope of this work. We can, however, find evidence in the literature that the hypothesis of strongly elongated magnetic turbulence, radially extending up to the plasma edge, is indeed well-founded. As an example, from figures 4 and 5 of [48], we note that the magnetic component of the turbulent fields exhibits strongly elongated features. Even more importantly, the radial extension of the $A_{\|}$ perturbations is longer than that for the electrostatic potential. The same authors then published a second paper in 2011 [49] where nonlinear, multi-mode simulations at large plasma beta were presented. Results once again show that fluctuations in the parallel electromagnetic potential are rather elongated (see figure 3 in [49]). They do vanish close to the very edge of the plasma, although this is due to the particular boundary conditions $A_{\|}=0$ at $r / a=1$ imposed in the numerical scheme for computational reasons. It is easy to imagine that, if the code could be made to allow for fluctuations in $A_{\|}$of finite amplitude up to $r / a=1$, the electromagnetic component of the turbulent fluctuation spectra would reach the plasma boundary and the position of the magnetic sensors on the low-field side wall, as for instance presented in $[50,51]$.

Figures 8 to 10 show the magnetic fluctuation measurements for the three discharges in the alpha-heating scan selected for figure 3 , illustrating the full range of variation in the DT ratio and $P_{\alpha \mathrm{FUS}}$, namely \#41069 (figure 8) with $n_{\mathrm{T}} /\left(n_{\mathrm{D}}+n_{\mathrm{T}}\right)=0$ and $\left\langle n_{\alpha} / n_{\mathrm{e}}\right\rangle=0$, \#42856 (figure 9) with $n_{\mathrm{T}} /\left(n_{\mathrm{D}}+n_{\mathrm{T}}\right)=0.53$ and $\left\langle n_{\alpha} / n_{\mathrm{e}}\right\rangle=0.0352$, and 

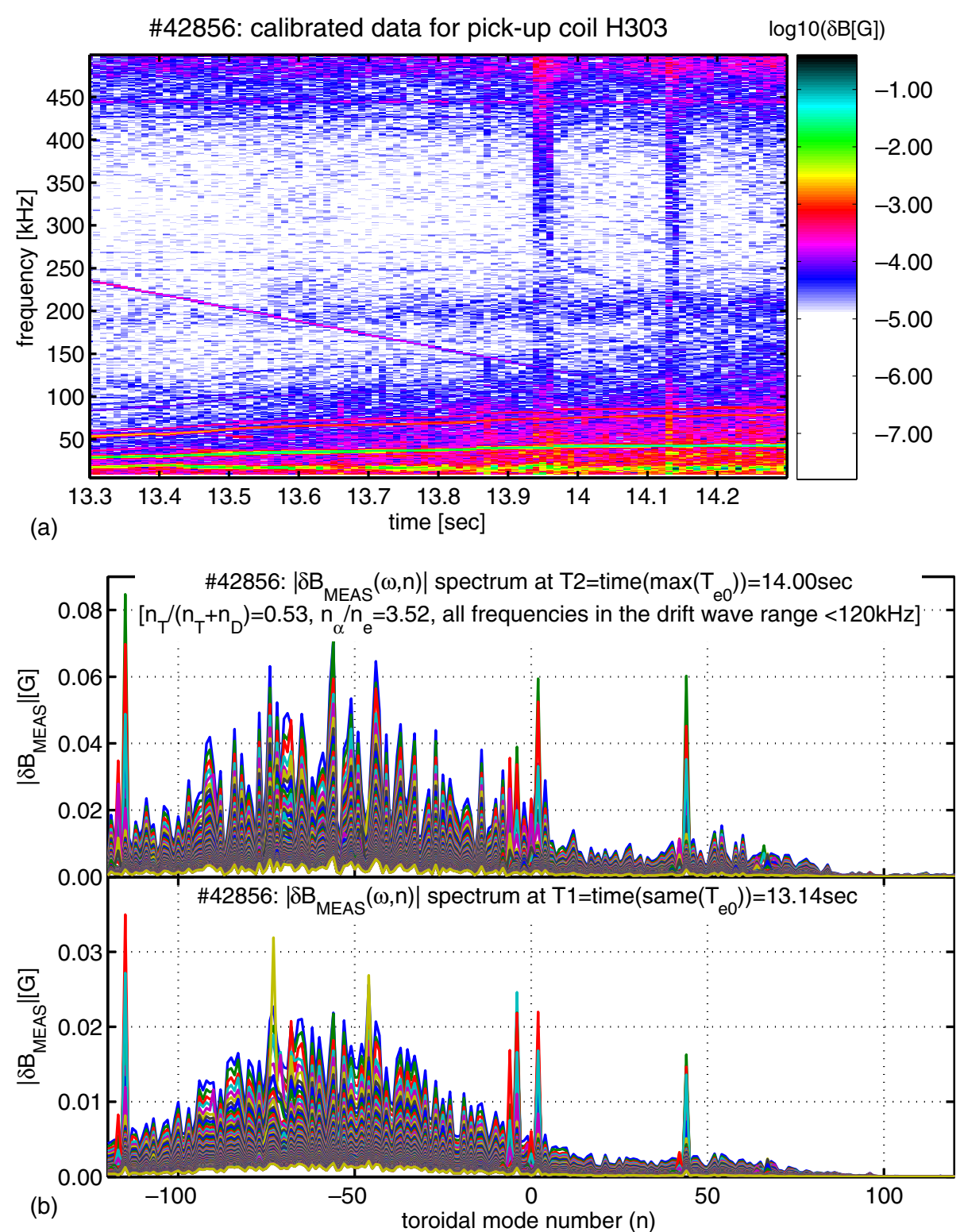

Figure 9. Measured magnetic fluctuation data for \#42856. Top frame: time/frequency spectrogram; bottom frames: auto-power spectrum at the time points $\mathrm{T} 1\left(\equiv \operatorname{same}\left(T_{\mathrm{e} 0}\right)\right)$ and $\mathrm{T} 2\left(\equiv \max \left(T_{\mathrm{e} 0}\right)\right)$.

\#43011 (figure 10) with $n_{\mathrm{T}} /\left(n_{\mathrm{D}}+n_{\mathrm{T}}\right)=0.92$ and $\left\langle n_{\alpha} / n_{\mathrm{e}}\right\rangle=$ 0.0107. In all of these figures we show in the top frame the spectrogram from one of the magnetic channels (the pick-up coil $\mathrm{H} 303$ ) acquired by the $\mathrm{KC} 1 \mathrm{~F}$ diagnostic system in the full frequency range of the calibrated measurements, extending from 10 up to $500 \mathrm{kHz}$, and calculated over the time window of interest $(13.30 \leqslant$ time $(s) \leqslant 14.30)$. In the bottom frames we show the auto-power spectrum of the toroidal components of the measured magnetic field amplitude $\left|\delta B_{\text {MEAS }}(\omega, n)\right|$ for the fluctuation spectra evaluated using the SRS method in the drift-wave frequency range at the time points T1 and T2. The $\left|\delta B_{\text {MEAS }}(\omega, n)\right|$ auto-power spectrum is obtained by combining all the available $\mathrm{KC} 1 \mathrm{~F}$ and CATS channels acquiring magnetic data from pick-tip coils located at the same poloidal angle (13 sensors in total, non-uniformly distributed along the toroidal angle direction). Finally, note that in the bottom frames of figures $8-10$, the different traces overlaid on top of each other correspond to the different timebin frequencies, the frequency interval between traces being $\sim 500 \mathrm{~Hz}$ (i.e. $490 \mathrm{kHz}$ subdivided in 1024 FFT points), with a $1 \mathrm{~ms}$ binning time. Remember also that in these frames we have not yet removed the components associated with low$|n|<10$ coherent modes, such as NTMs, sawteeth and ELMs, faulty digitizer channels, corresponding to some of the high$|n|>50$, and to the saddle coil signal, and that components with $|n|>100$ are unlikely to be related to physical modes.

From the top frames in figures 8-10, we note that in the frequency range up to $500 \mathrm{kHz}$ no coherent fast-ion driven modes, such as TAEs $(\sim 150 \mathrm{kHz})$ and elliptical AEs $(\sim 300 \mathrm{kHz})$, are observed. Furthermore, in all the discharges in the alpha-heating experiment low- $n$ NTMs were present with similar amplitudes in the low-frequency range, between $\sim 10$ and $\sim 35 \mathrm{kHz}$. The triangular sweeping waveform seen in figure 9 for \#42856 is the signal from the saddle coils, used 

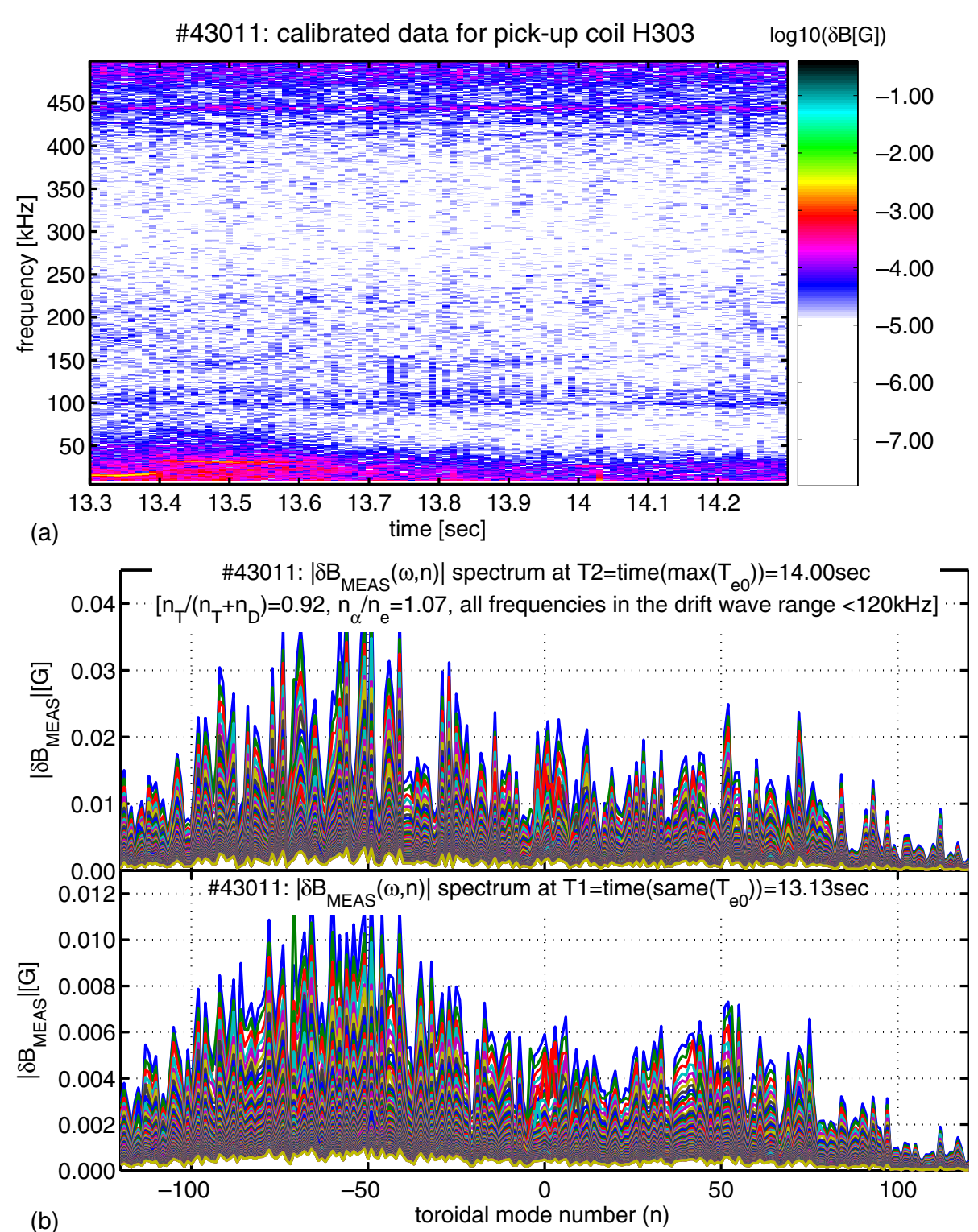

Figure 10. Measured magnetic fluctuation data for \#43011. Top frame: time/frequency spectrogram; bottom frames: auto-power spectrum at the time points $\mathrm{T} 1\left(\equiv \operatorname{same}\left(T_{\mathrm{e} 0}\right)\right)$ and $\mathrm{T} 2\left(\equiv \max \left(T_{\mathrm{e} 0}\right)\right)$.

to measure the damping rate of TAEs in this discharge [52]. Hence, as previously reported in [4], the presence or absence of coherent, current/pressure-driven (low-frequency) and higher frequency fast-ion driven modes can be discounted to explain the anomalous ion heating observed with the $\alpha$ s. Note also that the spectral amplitude of the different frequency components is actually very much reduced and becomes almost negligible above $\sim(70 \pm 20) \mathrm{kHz}$.

Now focussing our attention to the data presented in the middle and bottom frames of figures 8-10, we find that ITG modes and TEMs effectively coexist over the same toroidal mode number domain, in the range $25<|n|<100$, which is broadly consistent with the results of the GENE simulations. Second, the amplitude of the peaks appearing in these spectra for positive toroidal mode numbers, corresponding to incoherent turbulence in the ion-drift direction, decreases significantly from \#41069 (figure 8, the lowest $n_{\mathrm{T}} /\left(n_{\mathrm{D}}+n_{\mathrm{T}}\right)$ point in the DT mixture ratio scan) to \#42856 (figure 9), which has the highest value of $\left\langle n_{\alpha} / n_{\mathrm{e}}\right\rangle=0.0352$ and $P_{\alpha \mathrm{FUS}}$. Third, we find from visual inspection that the frequencyintegrated signal in the drift-wave range for positive toroidal mode numbers decreases from \#41069 to \#42856, but then increases again to \#43011 (figure 10, the highest $n_{\mathrm{T}} /\left(n_{\mathrm{D}}+n_{\mathrm{T}}\right)$ point in the DT mixture ratio scan). Conversely, the frequencyintegrated signal in the drift-wave range for negative toroidal mode numbers, corresponding to incoherent turbulence in the electron channel, does not appear to vary much across the DT mixture ratio scan. Note also that the data presented in the bottom frame of figure 10 for \#43011 (where we had the largest value of $n_{\mathrm{T}} /\left(n_{\mathrm{D}}+n_{\mathrm{T}}\right)=0.92$ and a small value of $\left.\left\langle n_{\alpha} / n_{\mathrm{e}}\right\rangle=0.0107\right)$ have a much larger tail for negative toroidal mode numbers $n<-80$ than that obtained for \#41069 (where we had $n_{\mathrm{T}} /\left(n_{\mathrm{D}}+n_{\mathrm{T}}\right)=0$ and $\left\langle n_{\alpha} / n_{\mathrm{e}}\right\rangle=0$ ) and for \#42870 (where we had $n_{\mathrm{T}} /\left(n_{\mathrm{D}}+n_{\mathrm{T}}\right)=0.19$ and $\left.\left\langle n_{\alpha} / n_{\mathrm{e}}\right\rangle=0.0134\right)$. As the density of $\alpha \mathrm{s}$ is very similar in these discharges, this observation suggests an isotopic 
effect of the bulk plasma (i.e. not related to the presence of $\alpha$ s) on the turbulence spectrum, particularly in the electron channel.

These observations on the change of the turbulence spectra as a function of the density of $\alpha$ s can be cast in a more quantitative form by plotting the frequency-integrated signal in the drift-wave range $\int\left|\delta B_{\text {MEAS }}(\omega, n)\right| \mathrm{d} \omega$. These data are shown for all the discharges in the alpha-heating scan at the time-point $\mathrm{T} 1$ in figure $11(a)$ and at the timepoint $\mathrm{T} 2$ in figure $11(b)$, respectively, this time keeping only the turbulent components (again, remember that spectral components with $|n|>100$ are unlikely to be related to physical modes). The horizontal error bar shows the accuracy on the $n$-number determination via the SRS method, which is related to the spectral window of the 13 magnetic sensors used for this analysis. The vertical error bar indicates the accuracy on the frequency-integral over the drift-wave range $\int \operatorname{err}\left(\left|\delta B_{\text {MEAS }}(\omega, n)\right|\right) \mathrm{d} \omega$, which is related to the error on each individual toroidal component of the spectrum calculated using the SRS method.

Comparing figure 11(a) with figure 11(b), we first note that (with the notable exception of \#43011, as indicated previously) the shape of the integrated $\int\left|\delta B_{\text {MEAS }}(\omega, n)\right| \mathrm{d} \omega$ signal for negative toroidal mode numbers is very similar at the time-point $\mathrm{T} 2$ of the maximum in $T_{\mathrm{e} 0}$ (figure $11(b)$ ) and at the time-point $\mathrm{T} 1$ of the same $T_{\mathrm{e} 0}$ (figure $11(a)$ ). This clearly indicates that the turbulence in the electron-drift direction is not much affected by the presence of the $\alpha$ s, with only perhaps an isotopic effect due to the bulk plasma composition. For the data taken at the time-point T1 (same value of $T_{\mathrm{e} 0}$, figure $11(a)$ ), the turbulence in the ion-drift direction (positive toroidal mode numbers) has smaller amplitudes (by approximately $\sim 40 \%$ ) and a narrower spread (spectra centred around $n \sim 60$ with a variance $\sim 15$ for $\left\langle n_{\alpha} / n_{\mathrm{e}}\right\rangle \sim 0.03$ compared with a variance $\sim 25$ for $\left\langle n_{\alpha} / n_{\mathrm{e}}\right\rangle \sim 0.01$ ) when increasing the density of the $\alpha$ s above a critical value, as shown by comparing the three subplots in figure 11(a). These experimental observations on ITG and TEM turbulence are broadly in agreement with the results of our GENE simulations reported in section 3 .

This observation is consistent with the theoretical predictions by Angioni and Peeters (see figure 1 and the relevant discussion in [28]). However, in [28] the role of tracer particles is discussed, whereas with our GENE simulations we have shown that fusion-born $\alpha$ s cannot always be considered as a tracer species. We also note that Tardini and his colleagues have similarly linked the formation of an ion transport barrier on the ASDEX-Upgrade tokamak to the suppression of ITG turbulence due to thermal ion dilution by the slowing-down population of fast NBI ions [53]. However, in our GENE simulations we have also included a fastion energy dependence on the ITG stabilization mechanism which is derived from the turbulence measurement, thus differentiating our work from that of [53]. For the data taken at the time-point T2 (maximum value of $T_{\mathrm{e} 0}$, figure $11(\mathrm{~b})$ ), the turbulence in the ion-drift direction (positive toroidal mode numbers) has larger amplitudes (by approximately a factor $\sim 2$ ) and a larger spread (spectra centred around $n \sim$ 60 with a variance $\sim 35$ ) than at the time-point $\mathrm{T} 1$ (the same $\left.T_{\mathrm{e} 0}\right)$.
Finally, figures 12 and 13 show two different summary representations for the turbulence data for the alpha-heating experiment. In figure 12 we show the spectral components of the frequency-integrated signal $\int\left|\delta B_{\text {MEAS }}(\omega, n)\right| \mathrm{d} \omega$ for the same three discharges of figure 3 , corresponding to the extreme in the isotope ratio scan, separately at the time points $\mathrm{T} 1$, with $\alpha \mathrm{s}$ not thermalized, and $\mathrm{T} 2$, with $\alpha$ s fully thermalized, plotted as a function of the toroidal mode number. In figure 13, we show the total signal $\left|\delta B_{\text {MEAS }}\right|=\left(\Sigma_{n}\left(\int\left|\delta B_{\text {MEAS }}(\omega, n)\right| \mathrm{d} \omega\right)^{2}\right)^{1 / 2}$, plotted as a function of the alpha particle concentration $n_{\alpha} / n_{\mathrm{e}}$, separately for the ITG $\left(\Sigma_{n}\right.$ for $\left.n>20\right)$ and TEM $\left(\Sigma_{n}\right.$ for $\left.n<-20\right)$ channels, again at the two time points $\mathrm{T} 1$ and $\mathrm{T} 2$. We find that the presence of a minority population of highenergy, fusion-born $\alpha$ s not yet fully thermalized and above a critical density stabilizes the turbulence in the ion-drift direction, but practically does not affect the turbulence in the electron-drift direction. This experimental observation is again broadly consistent with the results of our GENE simulations. When the $\alpha$ s have sufficiently slowed down so that $T_{\mathrm{e} 0}$ and $T_{\mathrm{i} 0}$ have increased above the value obtained under similar plasma conditions but without the $\alpha \mathrm{s}$, the turbulence spectrum in the ion-drift direction reappears at somewhat larger amplitudes. This reappearance of the turbulence in the ion-drift direction occurs with the experimentally observed saturation in the ion temperature increase. Hence, we suggest this phenomenological mechanism as an explanation for the anomalous ion heating observed in the DTE1 experiment, namely that the initial stabilization of ITG turbulence due to a sufficiently large fraction of fusion-born $\alpha$ s that have not yet fully thermalized allows an initial increase in $T_{\mathrm{i}}$ above the level expected in these discharges, which in turn drives the ITG turbulence to reappear at a later stage in the discharge at higher levels than that observed in similar discharges without the $\alpha \mathrm{s}$.

We now need to reproduce, with GENE simulations, the measured reappearance of ITG modes as both ion and electron temperatures increase. Hence, we focus on the $T_{\mathrm{i} 0} / T_{\mathrm{e} 0}$ parameter, the quantity which varies the most during the alphaheating experiment, and take the discharge \#42856, which has the largest $T_{\mathrm{i} 0} / T_{\mathrm{e} 0}$ span, as an example of this analysis. A series of GENE simulations is once again performed allowing us to reconstruct the time evolution of the ITG and TEM growth rates, as illustrated in figure 14. The 'simulated' value for the intensity of ITG modes, as estimated from the value of the growth rate $\gamma_{\mathrm{ITG}}$, drops as the $\alpha$ s start to thermalize over the bulk plasma, from $t=12 \mathrm{~s}$ to $t=12.5 \mathrm{~s}$. The initial stabilization of the ITG modes, mainly obtained with beta effects and $\alpha \mathrm{s}$, translates into a different behaviour of the $T_{\mathrm{i}}$ and $T_{\mathrm{e}}$ profiles. The increased $T_{\mathrm{i}} / T_{\mathrm{e}}$ reinforces the turbulence which, once $\alpha$ s are thermalized and do not contribute any further to ITG stabilization, reverts back to the levels associated with the pre-NBI phase. This means that $\gamma_{\text {ITG }}$ gets back to the values obtained at the start of the NBI heating phase only around $t=13.4 \mathrm{~s}$. The overall dynamical evolution of $\gamma_{\text {ITG }}$ reproduces very well the measured value $\mid \delta B_{\text {MEAS }}($ ITG) $\mid$. This mechanism then allows the ion temperature to increase above the electron temperature over a time scale $\tau_{\mathrm{Ri}} \sim 0.8 \mathrm{~s}$, which is much faster than the alpha particle slowing-down time on the ions and the electron-ion energy equipartition time, which are all of the same order ( $4 \mathrm{~s}$ to $5 \mathrm{~s}$ ). During this time 

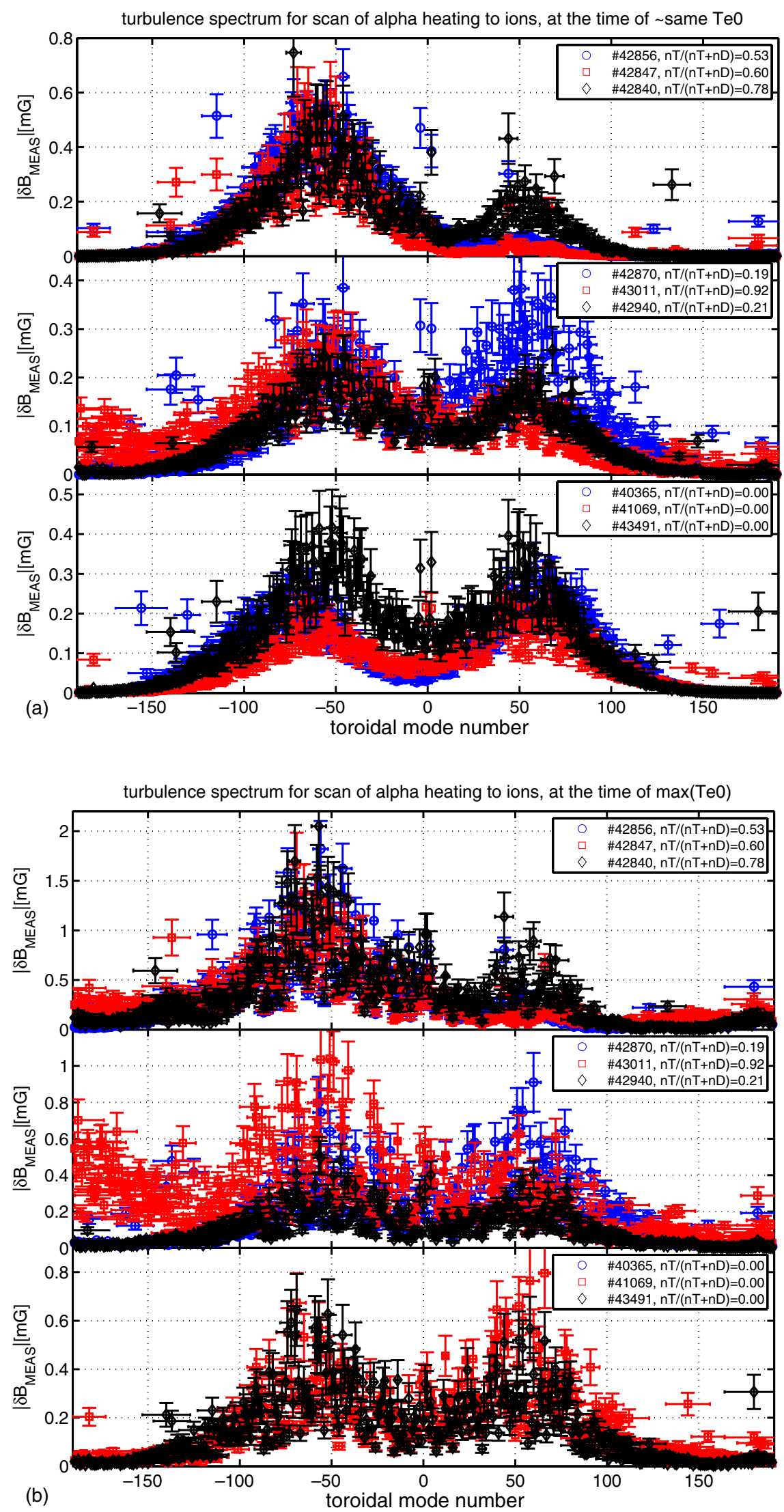

Figure 11. (a) Spectrum of the magnetic turbulence for all the nine discharges in the alpha-heating scan, integrated over the drift-wave frequency range at the time-point $\mathrm{T} 1$, corresponding to the same $T_{\mathrm{e} 0}$ for these discharges. $(b)$ Spectrum of the magnetic turbulence for all the nine discharges in the alpha-heating scan, integrated over the drift-wave frequency range at the time-point T2, corresponding to the maximum in $T_{\mathrm{e} 0}$ for these discharges. 


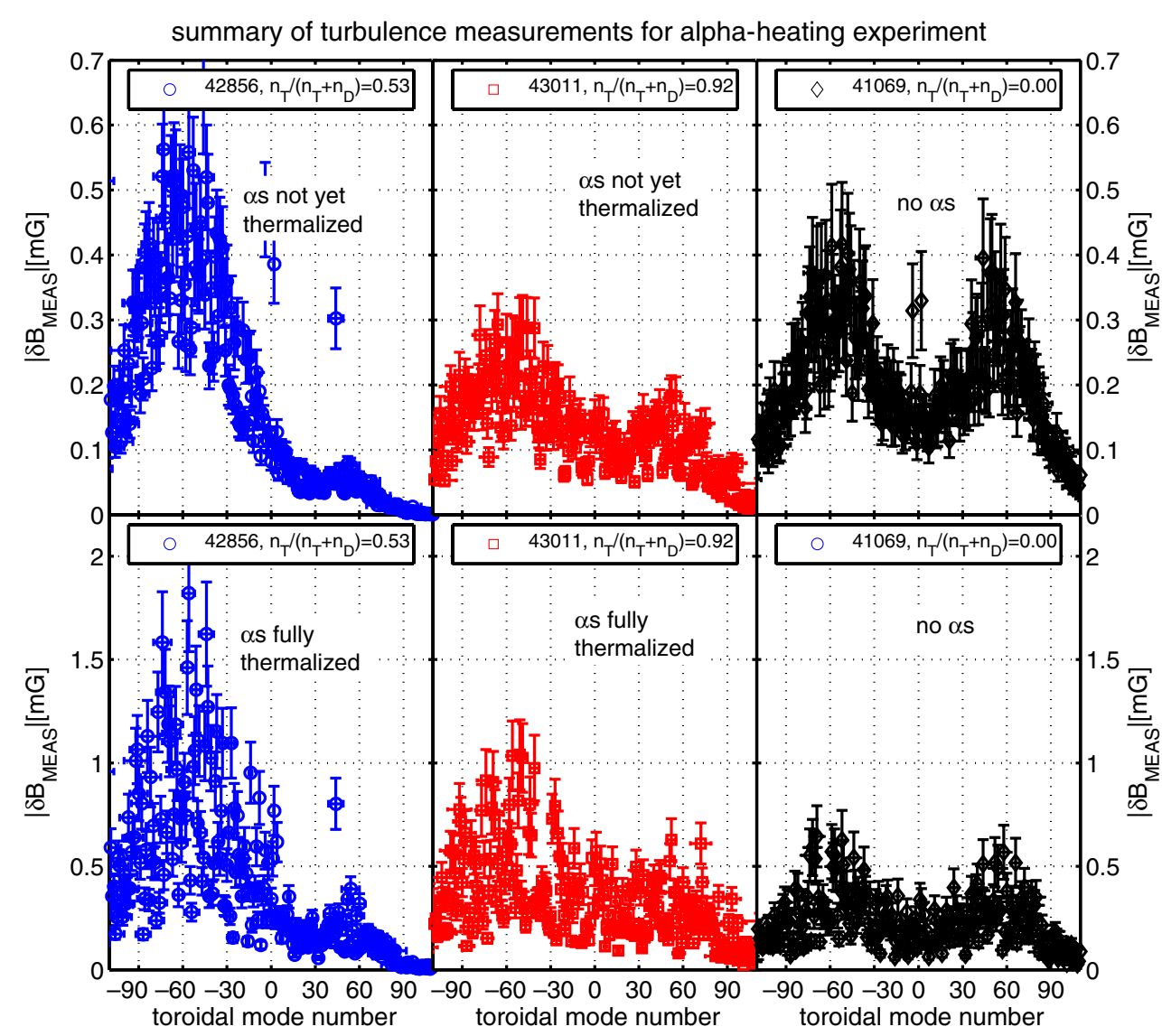

Figure 12. Summary of the turbulence spectral data for the alpha-heating experiment, shown as a function of the toroidal mode number for the same discharges of figure 3 , corresponding to the extreme in the $n_{\mathrm{T}} /\left(n_{\mathrm{D}}+n_{\mathrm{T}}\right)$ ratio isotope ratio scan. The data are shown separately for the two time points T1 (top frames: $\alpha$ s not thermalized, time-point with the same $T_{\mathrm{e} 0}$ ) and T2 (bottom frames: $\alpha \mathrm{s}$ fully thermalized, time-point corresponding to the maximum in $\left.T_{\mathrm{e} 0}\right)$.

window the ratio $T_{\mathrm{i} 0} / T_{\mathrm{e} 0}$ continuously increases, and reaches its maximum value $T_{\mathrm{i} 0} / T_{\mathrm{e} 0} \approx 1.8$ at around $t=13.35 \mathrm{~s}$. An increase in $\gamma_{\text {ITG }}$ above the value observed in the preheating phase, hence in the ITG micro-instability strength, is then observed to occur from $t=13.7 \mathrm{~s}$ onwards, after around one alpha particle slowing-down time on the electrons, $\tau_{\alpha \mathrm{e}} \sim 1.2 \mathrm{~s}$, i.e. when the fusion-born $\alpha$ s have had the time to fully thermalize so that their mean energy has decreased sufficiently. This, in turn, causes a reduction in the ion temperature and in the $T_{\mathrm{i} 0} / T_{\mathrm{e} 0}$ ratio, despite the continuous injections of NBI ions collisionally transferring their energy to thermal ions, whereas the electron temperature continues to increase, both features being clearly observed experimentally. Conversely, the behaviour of TEM, as deduced from the time evolution of $\gamma_{\mathrm{TEM}}$ and $\left|\delta B_{\mathrm{MEAS}}(\mathrm{TEM})\right|$, remains essentially unaffected throughout the $\alpha$ s' thermalization process, as $\gamma_{\text {TEM }}$ and $\mid \delta B_{\text {MEAS }}$ (TEM) $\mid$ are almost constant throughout this phase. Again, the measurements and simulations of ITG and TEM turbulence are broadly in agreement. It is important to note here that, as we take the actual evolution of the background plasma parameters as input for these GENE calculations, we are effectively supplementing the intrinsic role of the $\alpha \mathrm{s}$ with their feedback action on the $T_{\mathrm{i}} / T_{\mathrm{e}}$ ratio via the suppression of the ITG turbulence.

\section{Why anomalous ion heating was not observed in other DT experiments?}

The simulation results and the measurements reported in sections 3 and 4, respectively, have provided a phenomenological explanation for the anomalous ion heating observed in the JET alpha-heating experiment of 1997, which reached a peak fusion energy gain $Q_{\mathrm{DT}} \approx 0.65$ and peak fusion power $P_{\mathrm{FUS}} \approx 16 \mathrm{MW}$, maintained for over half an energy confinement time. It is now important to understand [54] why no anomalous ion heating was observed in the DT experiments performed in the Tokamak Fusion Test Reactor (TFTR) [17] from 1993 to 1997 (which reached a peak $Q_{\mathrm{DT}} \approx$ 0.25 [18]), neither in the Preliminary Tritium Experiment (PTE) of JET in 1992 (reaching $Q_{\mathrm{DT}} \approx 0.15$ [55]) or with the other high-performance operational scenarios used for the JET DT experiments in 1997 [1, 10, 56, 57]. A simple, quantitative answer to these questions is readily obtained using the theoretical framework presented in $[58,59]$. A fluid treatment of ITG turbulence predicts that it becomes locally unstable when the ratio of the density to the ion temperature scale length $\eta_{\mathrm{i}}=L_{n} / L_{T_{\mathrm{i}}}$ is above the marginal stability value $\eta_{\mathrm{iC}}=\left(L_{n} / L_{T_{i}}\right)_{\mathrm{CRIT}}$

$$
\frac{L_{n}}{L_{T_{\mathrm{i}}}} \geqslant\left(\frac{L_{n}}{L_{T_{\mathrm{i}}}}\right)_{\mathrm{CRIT}}=\frac{4}{3}\left(1+\frac{T_{\mathrm{i}}}{T_{\mathrm{e}}}\right)\left(1+\frac{r}{q^{2}} \frac{\mathrm{d} q}{\mathrm{~d} r}\right) \frac{L_{n}}{R}=\eta_{\mathrm{iC}},
$$




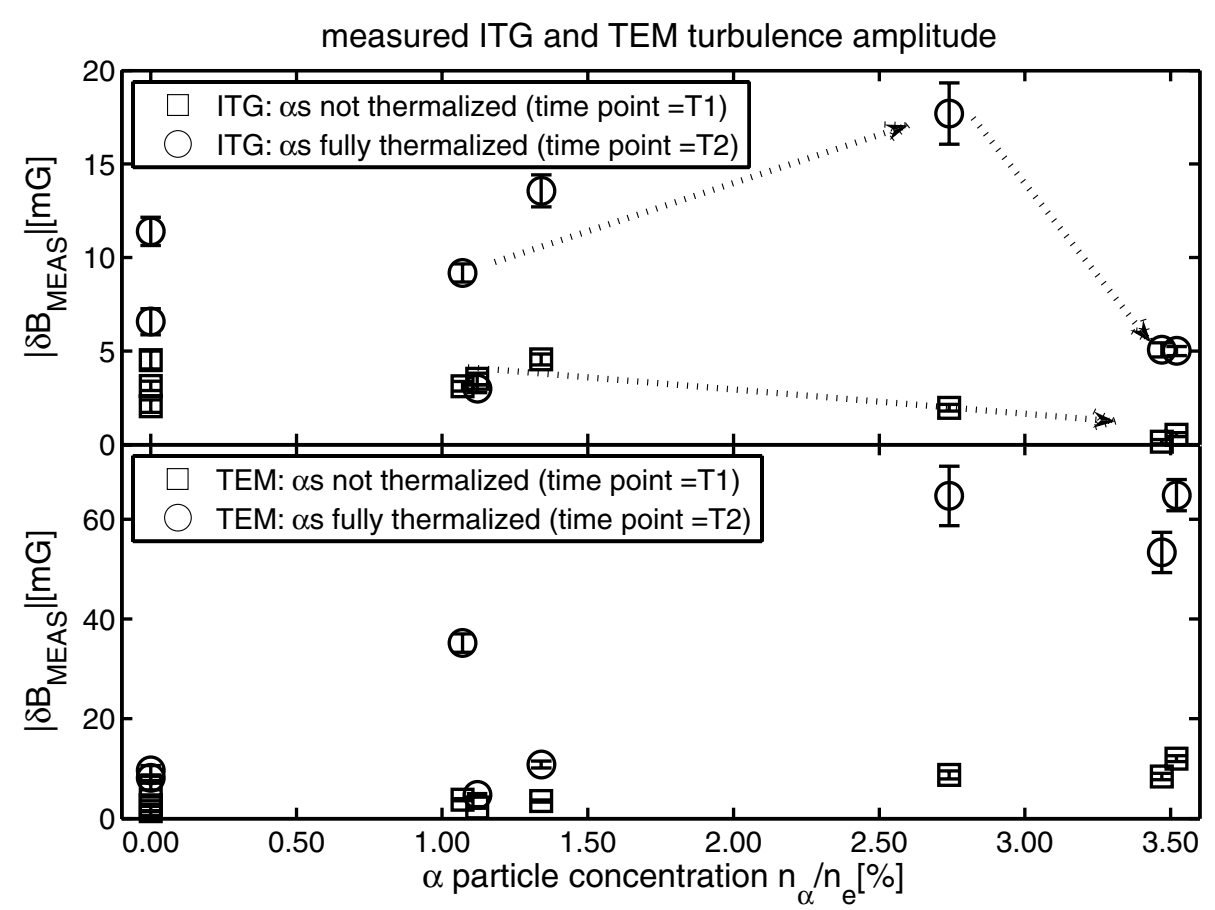

Figure 13. Summary of the measured turbulent spectra $\left|\delta B_{\text {MEAS }}\right|$ for the nine discharges in the alpha-heating experiment, shown separately for the ion (ITG: top frame) and electron (TEM: bottom frame) drift-wave channels, for the two time points T1 ( $\alpha$ s not thermalized) and T2 ( $\alpha$ s fully thermalized), and plotted as a function of the alpha particle concentration $n_{\alpha} / n_{\mathrm{e}}$. In the top frame, lines are drawn to guide the eyes.

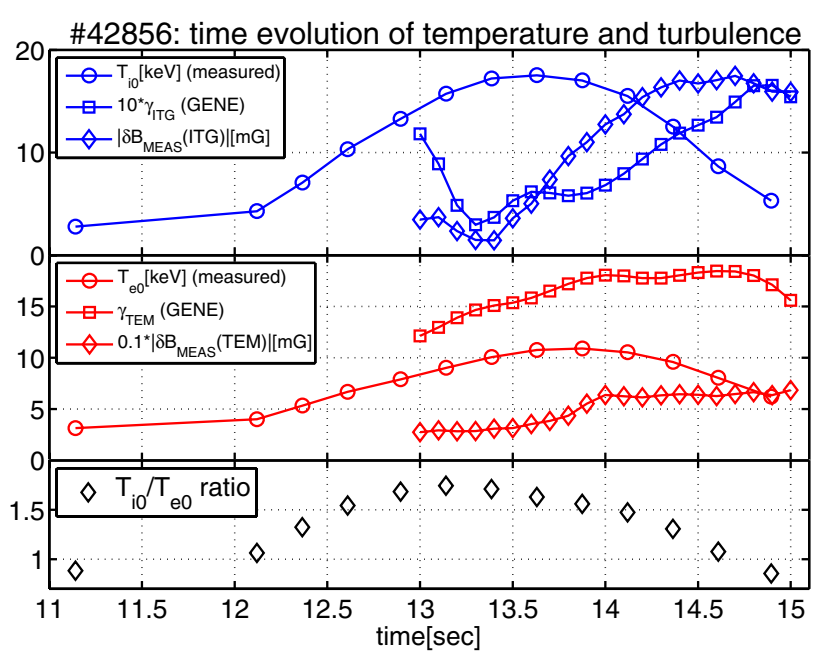

Figure 14. Reconstructed time evolution of the TEM and ITG turbulence amplitude and growth rates for \#42856 plotted as a function of the background ion and electron temperatures in the plasma core, and their ratio $T_{\mathrm{i} 0} / T_{\mathrm{e} 0}$.

when the density scale length is above a critical value $\left(L_{n} / R\right)_{\mathrm{CRIT}}$

$$
\frac{L_{n}}{R} \geqslant\left(\frac{L_{n}}{R}\right)_{\mathrm{CRIT}}=0.9\left(1+\frac{T_{\mathrm{i}}}{T_{\mathrm{e}}}\right)^{-1}\left(1+\frac{r}{q^{2}} \frac{\mathrm{d} q}{\mathrm{~d} r}\right)^{-1} .
$$

In equations (2) and (3) $q$ is the safety factor profile, and the radial coordinate $r$ has been dropped for simplicity in all the profile quantities (i.e. $X \equiv X(r)$ ). Figure 15 shows an example of this fluid treatment of the ITG marginal stability criterion for discharge \#42856, where all quantities entering equations (2) and (3) have been weighted over the ITG eigenfunction shown

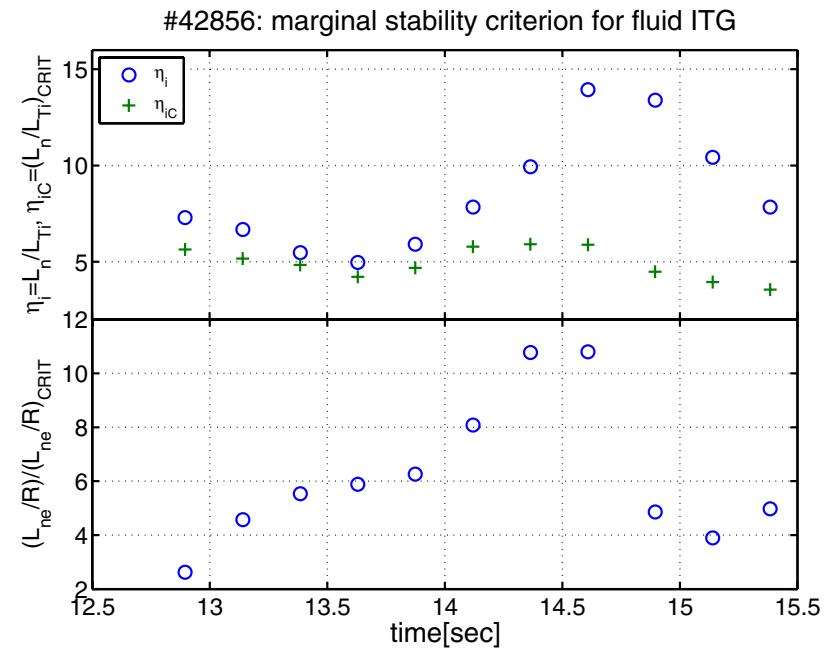

Figure 15. Marginal stability criterion for the fluid ITG for discharge \#42856, weighted over the ITG eigenfunction shown in figure $7(a)$. Note that from time $=14 \mathrm{~s}$, with fully thermalized $\alpha \mathrm{s}$, the ratio of the density to the temperature scale length $\left(\eta_{\mathrm{i}}\right)$ starts to diverge from the critical value $\left(\eta_{\mathrm{iC}}\right)$ that determines the stability limit, whereas for $13<\operatorname{time}(s)<14$, with $\alpha$ s close to their birth energy, we have that $\eta_{i}$ is just slightly above $\eta_{\mathrm{ic}}$. This implies that from time $=14 \mathrm{~s}$ ( $\equiv \mathrm{T} 2$ in the turbulence simulations and measurements) it is more difficult for the (fully thermalized) $\alpha$ s to stabilize ITG turbulence, whereas before time $=14 \mathrm{~s}(\equiv \mathrm{T} 1$ in the turbulence simulations and measurements) a relatively minor contribution from (non-thermal) $\alpha$ s can effectively contribute to stabilization of ITG turbulence.

in figure $7(a)$. During the early phase of the NBI heating, for $13<\operatorname{time}(s)<14$, with $\alpha$ s close to their birth energy, the plasma profiles for the JET alpha-heating experiment have $\left(L_{n} / R\right)>\left(L_{n} / R\right)_{\mathrm{CRIT}}$ and $\eta_{\mathrm{i}} \sim 1.15 \eta_{\mathrm{iC}}$ : a small 
contribution from the $\alpha$ s can then have a substantial effect on $\gamma_{\text {ITG }}$. Conversely, from time $=14 \mathrm{~s}$, with fully thermalized $\alpha \mathrm{s}$, the ratio of the density to the temperature scale length $\eta_{\mathrm{i}}$ starts to diverge from the critical value $\eta_{\mathrm{iC}}$ determining the fluid ITG marginal stability limit, making it much more difficult for $\alpha$ s to contribute to ITG turbulence stabilization. This simple criterion provides a remarkably correct and even semi-quantitative understanding of why anomalous ion heating has been observed due to ITG turbulence suppression in the JET alpha-heating experiment of 1997.

The TFTR DT experiments [18] also had $\left(L_{n} / R\right)>$ $\left(L_{n} / R\right)_{\mathrm{CRIT}}$ but $\eta_{\mathrm{i}}>5 \eta_{\mathrm{iC}}$, very far away from the ITG marginal stability limit for any anomalous ion heating to occur with $\mathrm{a} \sim 1 \%$ concentration of $\alpha \mathrm{s}$. Similarly, the plasma profiles for JET PTE experiment [55] and for the other highperformance scenarios $[1,10,56,57]$ developed for the DTE1 campaign were such that $\left(L_{n} / R\right)>\left(L_{n} / R\right)_{\mathrm{CRIT}}$, but had $\eta_{\mathrm{i}} / \eta_{\mathrm{iC}}$ in the range $\eta_{\mathrm{i}} / \eta_{\mathrm{iC}} \approx 1.5$ (usually with $n_{\alpha} / n_{\mathrm{e}} \approx 0.01$ ) to $\eta_{\mathrm{i}} / \eta_{\mathrm{iC}} \approx 5$ (usually with $n_{\alpha} / n_{\mathrm{e}} \approx 0.03$ ). In fact, taking as an instructive example the discharges in the DTE1 campaign, an empirical criterion for ITG stabilization by fusion-born $\alpha$ s can be quantitatively formulated as follows:

$$
\frac{\eta_{\mathrm{i}} / \eta_{\mathrm{iC}}}{\left\langle n_{\alpha} / n_{\mathrm{e}}\right\rangle\left(T_{\alpha}(\mathrm{MeV}) / 3.5(\mathrm{MeV})\right)}<40
$$

where again for $T_{\alpha}$ we have taken the equivalent Maxwellian temperature of the $\alpha \mathrm{s}$ ' population as given by [27], $\left(L_{n} / R\right)>$ $\left(L_{n} / R\right)_{\mathrm{CRIT}}, 3.5 \mathrm{MeV}$ is the birth energy of the $\alpha \mathrm{s}$, and this criterion is valid locally or globally, depending on how the $\left\langle n_{\alpha} / n_{\mathrm{e}}\right\rangle$ average entering equation (4) is taken.

\section{Predictions on ITG stabilization by alpha particles for ITER}

In Science [60], Nature [61,62] and more recently in Scientific American [63], it has been shown that turbulence could negatively affect ITER operation by worsening energy confinement through increased heat and particle transport. Hence, it is important to assess whether this mechanism for ITG turbulence stabilization by fusion-born $\alpha$ s could also work in the foreseen ITER plasmas. For this analysis, we have performed a number of simulations using GENE for the ITER reference steady-state scenario [64,65], the so-called $\mathrm{S} 4$ baseline scenario. As in the analysis reported in [59], for the turbulence simulations presented here we have used $\beta_{\mathrm{e}}=$ $1.5 \%, k_{y} \rho_{\mathrm{s}}=0.6,\left(L_{n} / R_{0}\right)_{\mathrm{GENE}}=2$ and $\left(L_{T_{\mathrm{i}}} / R_{0}\right)_{\mathrm{GENE}}=$ $1 / 3.5\left(R_{0}=6.2 \mathrm{~m}\right.$ is the ITER major radius), and we have scanned the concentration and temperature of the $\alpha \mathrm{s}$ in the range $0 \leqslant n_{\alpha} / n_{\mathrm{e}} \leqslant 0.1$ and $80 \leqslant T_{\alpha} / T_{\mathrm{e}} \leqslant 160$, respectively.

Figure 16 shows the radial profile versus $\sqrt{\psi_{\mathrm{N}}}$ for the intended values for the main background plasma parameters for the S4 scenario during the flat-top phase for the plasma current $^{1}$. For comparison purposes, the data for the other ITER reference baseline scenarios S2 (inductive, plasma current $I_{\mathrm{p}}=15 \mathrm{MA}$ ), S3a (hybrid, $I_{\mathrm{p}}=9 \mathrm{MA}$ ) and S5 (inductive, $I_{\mathrm{p}}=17 \mathrm{MA}$ ) are also shown. All of these data have

1 ITER-organization, magnetic equilibria and plasma parameters for the reference scenarios [S2, S3a, S4 and S5], provided courtesy of G. Vayakis and G. Saibene. been computed using analytic fit to the magnetic equilibrium provided by ASTRA calculations [66]. The electron density profile is intended to be very flat up to $\sqrt{\psi_{\mathrm{N}}} \approx 0.85$, i.e. $L_{n} \gg R_{0}$, so that the condition $\left(L_{n} / R\right)>\left(L_{n} / R\right)_{\mathrm{CRIT}}$ of equation (3) is always satisfied for these four ITER baseline scenarios. The volume-averaged concentration of $\alpha \mathrm{s}$ is $\left\langle n_{\alpha} / n_{\mathrm{e}}\right\rangle_{\mathrm{S} 2}=2.87 \%,\left\langle n_{\alpha} / n_{\mathrm{e}}\right\rangle_{\mathrm{S} 3 \mathrm{a}}=0.71 \%,\left\langle n_{\alpha} / n_{\mathrm{e}}\right\rangle_{\mathrm{S} 4}=$ $2.64 \%$, and $\left\langle n_{\alpha} / n_{\mathrm{e}}\right\rangle_{\mathrm{S} 5}=4.11 \%$, respectively, and the value at mid-radius (i.e. $\left.\sqrt{\psi_{\mathrm{N}}} \equiv 0.5\right)$ is $\left(n_{\alpha} / n_{\mathrm{e}}\right)_{\mathrm{S} 2}=3.37 \%$, $\left(n_{\alpha} / n_{\mathrm{e}}\right)_{\mathrm{S} 3 \mathrm{a}}=1.04 \%,\left(n_{\alpha} / n_{\mathrm{e}}\right)_{\mathrm{S} 4}=2.47 \%$, and $\left(n_{\alpha} / n_{\mathrm{e}}\right)_{\mathrm{S} 5}=$ $4.15 \%$, respectively.

Figure 17 shows the values of $\left(L_{n} / R\right)_{\mathrm{CRIT}}$ and $\eta_{\mathrm{iC}}=$ $\left(L_{n} / L_{T_{i}}\right)_{\mathrm{CRIT}}$ evaluated using the reference plasma profiles for the ITER baseline scenarios (S2, S3a, S4 and S5) $[6,67]$ together with the values of $\left(L_{n} / R_{0}\right)_{\mathrm{GENE}}=2$ and $\left(L_{T_{\mathrm{i}}} / R_{0}\right)_{\mathrm{GENE}}=4$, hence $\left(L_{n} / L_{T_{\mathrm{i}}}\right)_{\mathrm{GENE}}=8$, used for the GENE ITG and TEM turbulence simulations for scenario S4. Throughout the entire plasma cross-section, we find that $\left(L_{n} / R_{0}\right)_{\mathrm{GENE}} \approx 4 \times\left(L_{n} / R\right)_{\mathrm{CRIT}}$ and $1.2<$ $\left(L_{n} / L_{T_{\mathrm{i}}}\right)_{\mathrm{GENE}} /\left(L_{n} / L_{T_{\mathrm{i}}}\right)_{\mathrm{CRIT}}<1.8$ for scenario $\mathrm{S} 4$, hence in a range which is not very different from the JET DTE1 experimental values. This makes it conceivable that a sufficiently high concentration of $\alpha \mathrm{s}$ could reduce the growth rate of ITG turbulence in the GENE simulations. As the intended $L_{n} / R \rightarrow \infty$ up to $\sqrt{\psi_{N}} \approx 0.85$, i.e. over most of the plasma volume, to evaluate the critical $\left(\eta_{\mathrm{iC}}\right)_{\mathrm{ITER}}$ quantity plotted in figure 17, we have used in equation (2) for $L_{n} / R$ the value $L_{n} / R \equiv\left(R_{0} / R\right) \times\left(L_{n} / R_{0}\right)_{\mathrm{GENE}}$ for consistency with the GENE runs for scenario S4. Therefore, in figure 17 $\left(\eta_{\mathrm{iC}}\right)_{\text {ITER }}$ is then simply defined as

$$
\eta_{\mathrm{i}, \mathrm{C}}^{\mathrm{ITER}}(r)=\left(\frac{L_{n}}{L_{T_{\mathrm{i}}}}\right)_{\mathrm{CRIT}}^{\mathrm{ITER}} \equiv \frac{8}{3}\left(1+\frac{T_{\mathrm{i}}}{T_{\mathrm{e}}}\right)\left(1+\frac{r}{q^{2}} \frac{\mathrm{d} q}{\mathrm{~d} r}\right),
$$

where the notation $X \equiv X(r)$ has again been used for all the profile quantities as in equations (2), (3). Hence, consistently with this approach, we define the fluid ITG stability parameter for ITER $\left(\eta_{\mathrm{i}}\right)_{\mathrm{ITER}}$ as $\left(\eta_{\mathrm{i}}\right)_{\mathrm{ITER}}=\left(L_{n} / R_{0}\right)_{\mathrm{GENE}} \times\left(R_{0} / L_{T_{\mathrm{i}}}(r)\right)=$ $2 R_{0} / L_{T_{\mathrm{i}}}(r)$. Focussing specifically on the reference steadystate scenario $\mathrm{S} 4$, figure 18 shows the value of $\left(\eta_{\mathrm{i}}\right)_{\text {ITER }}$ evaluated using the reference plasma profiles and calculated with $L_{n} / R_{0}=2$ as in the GENE simulations. There is quite a significant difference between these two values, due to the intended density profile being flat up to $\sqrt{\psi_{\mathrm{N}}} \approx 0.85$, hence the ensuing choice of $L_{n} / R_{0}=2$ in GENE. We note, however, that these two estimates are very close to each other around midradius, where they reach a value $\eta_{\mathrm{i}} \sim 7 \times \eta_{\mathrm{iC}}$, much larger than the JET DTE1 case.

Finally, figure 19 shows the results for the ITG and TEM turbulence growth rate obtained in initial simulations performed with GENE for the ITER reference steady-state scenario S4. We do indeed find a reduction in $\gamma_{\mathrm{ITG}}$ as $n_{\alpha} / n_{\mathrm{e}}$ and $T_{\alpha} / T_{\mathrm{e}}$ increase, but whereas the $n_{\alpha} / n_{\mathrm{e}}$ dependence is similar to the JET DTE1 case, the $T_{\alpha} / T_{\mathrm{e}}$ dependence is not as favourable. This is due to $\eta_{\mathrm{i}} / \eta_{\mathrm{iC}} \sim 1.7$ and $\max \left(T_{\alpha} / T_{\mathrm{e}}\right) \sim 160$ with $T_{\mathrm{e}} \sim 25 \mathrm{keV}$ in these GENE simulations for the ITER baseline scenario $\mathrm{S} 4$, whereas $\eta_{\mathrm{i}} / \eta_{\mathrm{iC}} \sim 1.15$ and $\max \left(T_{\alpha} / T_{\mathrm{e}}\right) \sim 260$ with $T_{\mathrm{e}} \sim 10 \mathrm{keV}$ in JET. Hence, whereas the criterion for ITG stabilization by fusion-born $\alpha$ s given in equation (4) was satisfied for the JET DTE1 experiments, for the intended 


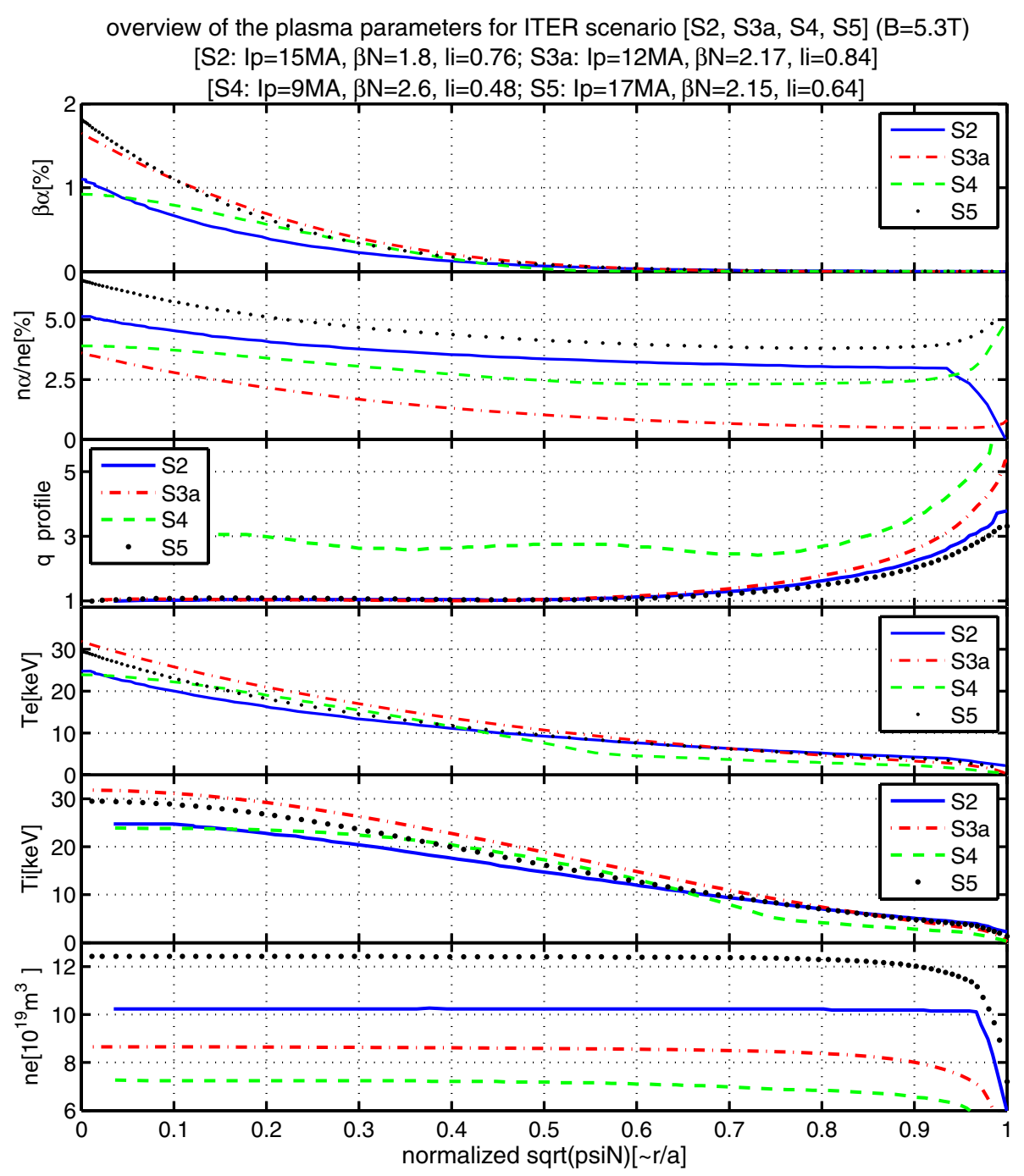

Figure 16. The main background plasma parameters for the ITER reference scenario [S2, S3a, S4 and S5] during the current flat-top phase; $\beta \alpha$ is the alpha particle normalized pressure profile and $n_{\alpha}$ is again the density of $\alpha$ s at their birth energy.

ITER S4 plasmas we have that $\left(\eta_{\mathrm{i}} / \eta_{\mathrm{iC}}\right) /\left(n_{\alpha} / n_{\mathrm{e}}\right) /\left(T_{\alpha}(\mathrm{MeV}) /\right.$ $3.5 \mathrm{MeV}) \sim 55$, i.e. about $30 \%$ above the empirical value of $\left(\eta_{\mathrm{i}} / \eta_{\mathrm{iC}}\right) /\left(n_{\alpha} / n_{\mathrm{e}}\right) /\left(T_{\alpha}(\mathrm{MeV}) / 3.5 \mathrm{MeV}\right)<40$ that has been found to be required for measurable ITG stabilization by fusion-born $\alpha \mathrm{s}$.

\section{Conclusions and an outlook for future experimental and modelling work}

Using a novel algorithm based on the sparse representation of signals to analyse the measured fluctuation spectra in the drift-wave frequency range for the discharges belonging to the alpha-heating experiment carried out during the JET DTE1 campaign of 1997, we find experimentally that the presence of a population of fusion-born $\alpha$ s that have not yet fully thermalized stabilizes the turbulence in the ion-drift direction, provided the concentration of the $\alpha$ s is sufficiently high, but practically does not affect the turbulence in the electron-drift direction. This allows the ion temperature to increase in magnitude above the value that could have been expected from neoclassical collisional slowing-down of the $\alpha \mathrm{s}$ on the electrons and electron-ion energy equipartition, this process occurring over a time scale much faster than those classically predicted. When the $\alpha$ s have sufficiently slowed down so that the electron and ion temperatures have increased above the value obtained under similar plasma conditions but without the $\alpha \mathrm{s}$, the turbulence spectrum in the ion-drift direction reappears at somewhat larger amplitudes, which in turn can be associated with the observed saturation and ensuing decrease in the ion temperature.

Simulations have been performed with the GENE code to analyse the predicted turbulent spectrum and the role of the plasma isotopic composition and of the $\alpha$ s themselves in its stabilization, and the results are qualitatively consistent with the phenomenology presented above. Using the ansatz that the computed growth rate for the ITG modes and TEMs can also give an indication of the spectral amplitude $\left|\delta B_{\text {MEAS }}(\omega, n)\right|$ of the measured turbulence, we find a very satisfying agreement between the turbulence measurements and the modelling with the local version of the GENE code with respect to the three key features of the observed phenomenology, namely (a) the dependence of the ITG stabilization on the density of the $\alpha$ s and their mean energy, (b) the independence of the amplitude of TEMs on the density of the $\alpha \mathrm{s}$ and their 
fluid ITG stability criteria for ITER operational scenarios [S2, S3a, S4, S5]

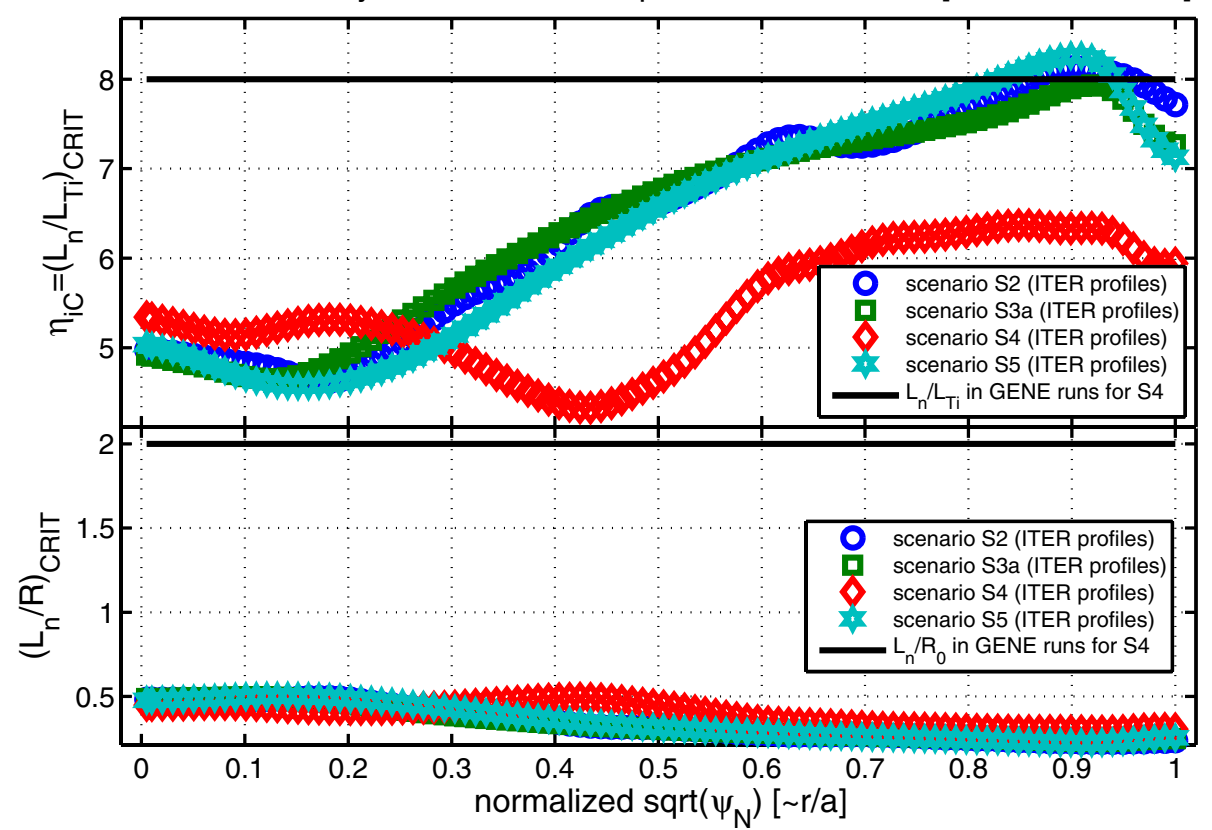

Figure 17. Evaluation of the fluid ITG stability parameters $\left(L_{n} / R\right)_{\mathrm{CRIT}}$ and $\eta_{\mathrm{iC}}=\left(L_{\mathrm{n}} / L_{T_{\mathrm{i}}}\right)$ CRIT for the ITER baseline scenarios [S2, S3a, $\mathrm{S} 4$ and S5], compared with the values for $\left(L_{n} / R_{0}\right)_{\mathrm{GENE}}=2,\left(L_{T_{\mathrm{i}}} / R_{0}\right)_{\mathrm{GENE}}=4$ and $\left(L_{n} / L_{T_{\mathrm{i}}}\right)_{\mathrm{GENE}}=8$ (the thick black horizontal lines in the two frames) used in the GENE simulations of ITG and TEM turbulence reported in section 6 for the baseline scenario S4.

GENE simulations:

fluid ITG stability parameters for ITER steady-state scenario S4

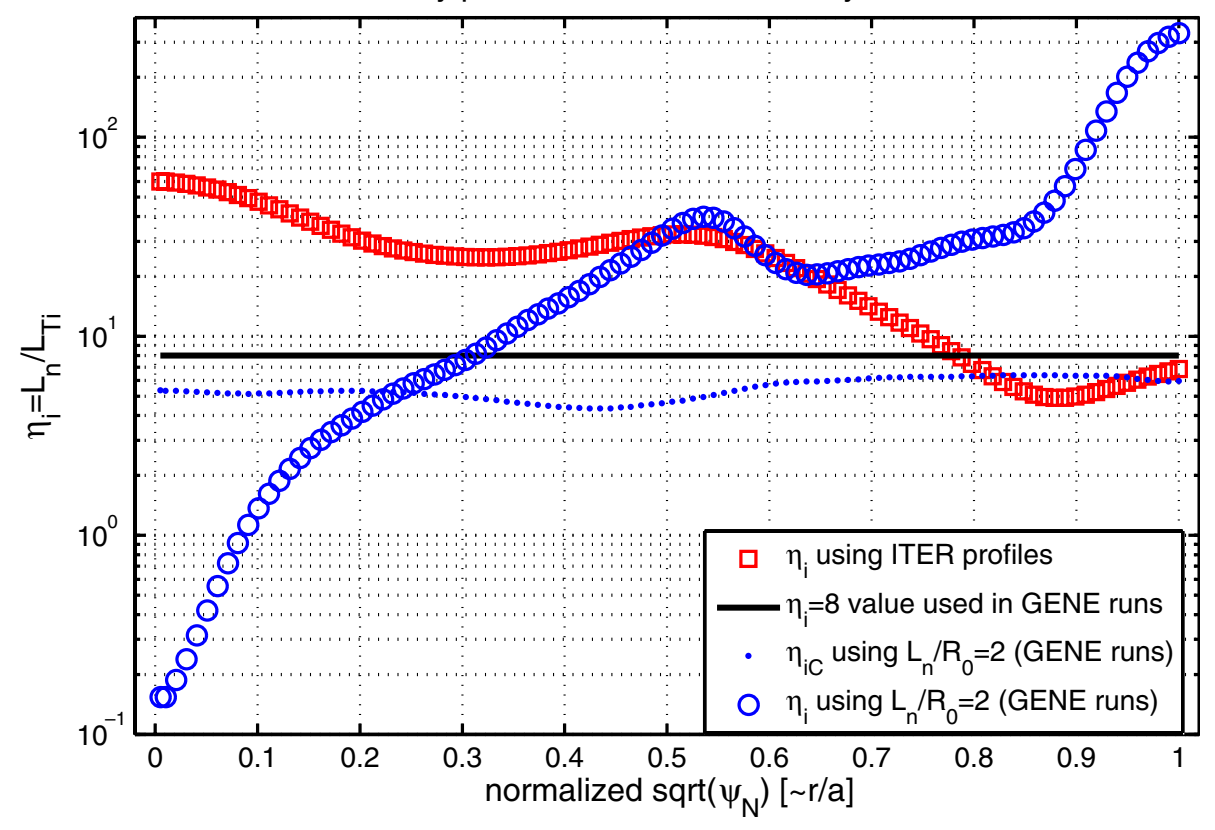

Figure 18. Comparison between the fluid ITG stability parameters $\left(\eta_{\mathrm{i}}\right)_{\text {ITER }}$ and $\left(\eta_{\mathrm{iC}}\right)_{\text {ITER }}$ evaluated using the nominal ITER profiles for the baseline scenario S4 and the values obtained with $L_{n} / R_{0}=2$ (GENE value); the thick black horizontal line indicates the actual value $\eta_{\mathrm{i}}=8$ used in the GENE simulations.

mean energy, and (c) the time evolution of the electron and ion temperatures as a function of the time evolution of the growth rate of ITG modes and TEMs. Finally, the radial position of the turbulent ITG eigenfunction indicated by crosscorrelation analysis corresponds to the region where previous TRANSP simulations indicated that a decrease in the ion thermal conductivity $\chi_{\mathrm{i}}$ was needed to explain the $T_{\mathrm{i}}$ increase. As ITG modes are responsible for ion heat and particle transport, we propose this direct stabilization of ITG turbulence by fusion-born $\alpha \mathrm{s}$ as a phenomenological explanation of the long standing puzzle that has been the anomalous ion heating observed in the JET DTE1 experiment of 1997. This 
ITG and TEM growth rate for ITER vs. alpha particle concentration: GENE simulations for steady-state reference scenario S4

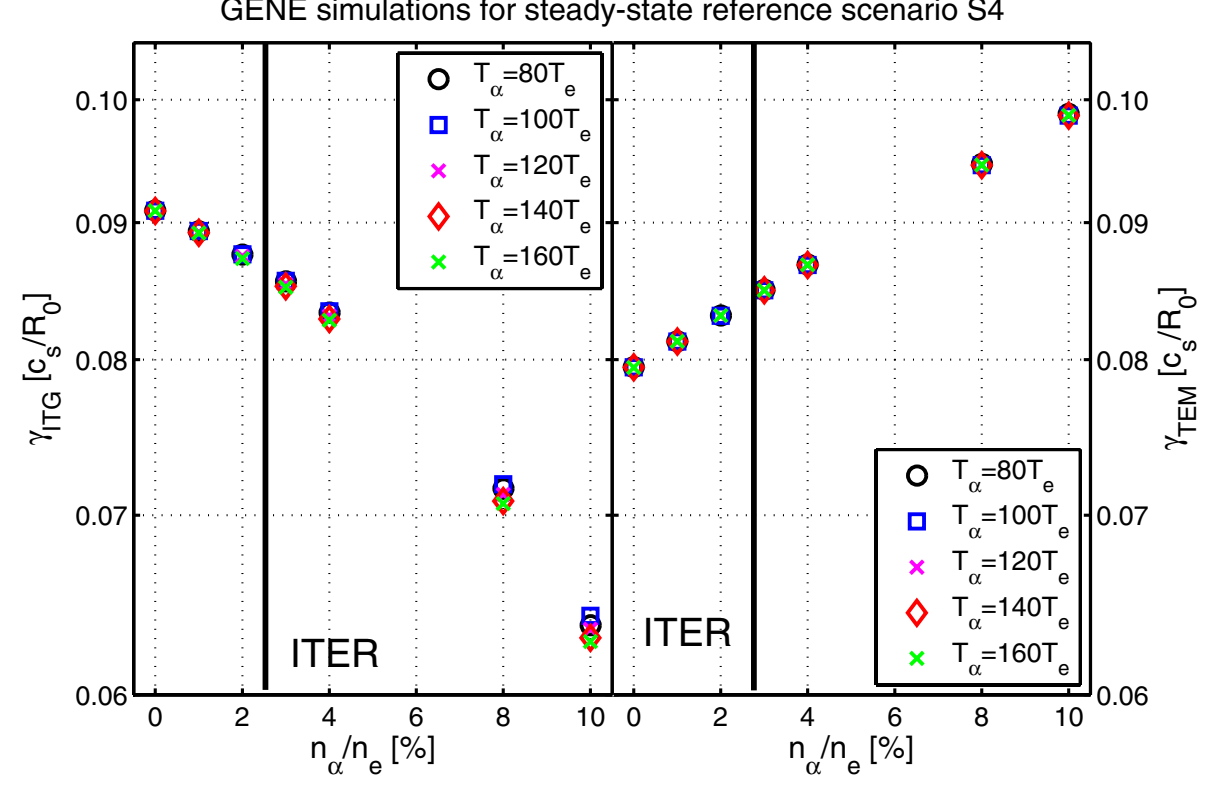

Figure 19. Predicted ITG and TEM growth rates for the ITER reference steady-state scenario S4, as calculated by GENE as a function of $n_{\alpha} / n_{\mathrm{e}}$ for different values of $T_{\alpha}$. The black vertical line at the $n_{\alpha} / n_{\mathrm{e}}=2.5 \%$ value represent the expected operational point for ITER S4 plasmas.

mechanism is a novel feature of our analysis, since it has never been previously considered, but other more well-known mechanisms, such as an isotopic effect, may also be at play, and will need to be included in further simulations aimed at obtaining a quantitative agreement between the predicted and measured ion temperature increase in these DTE1 discharges.

These results may open additional possibilities for future burning plasma experiments that aim at optimizing the path to ignition, as the current approach is to rely first on plasma preheating to increase the ion temperature to values that approach the optimal value of the fusion reactivity, then switch-off this pre-heating and rely on the fusion-born $\alpha$ s to collisionally heat the electrons during their slowing-down time, and then rely on the much slower process that is the electron-ion energy equipartition to keep the ion temperature close to that optimum value. We have demonstrated here that a much faster process exists for maintaining a sufficiently high ion temperature, whereby the fusion-born $\alpha$ s around their birth energy suppress the ITG turbulence if their concentration is above a certain critical value, this effectively causing a very beneficial reduction in the ion heat transport. Therefore, it may become possible to optimize the operational scenario of future burning plasmas such as ITER to take advantage of this one-step mechanism of ITG suppression and ion temperature increase in the presence of fusion-born $\alpha$ s.

To this end, an empirical criterion to evaluate whether ITG suppression by fusion-born $\alpha$ s can occur has been derived using JET and TFTR data. When this empirical criterion is applied to the ITER reference steady-state scenario, we find that ITG suppression by fusion-born $\alpha$ s can indeed occur, but not as efficiently as in the JET DTE1 case, as demonstrated using GENE simulations. The reason for this lies in the reference electron density profile being much too flat in comparison to the reference ion temperature profile. Hence, slight modifications to the background plasma profiles should allow ameliorating the stabilizing effect of the $\alpha$ s on the ITG turbulence in ITER. Additionally, it is important to remember that the physics of energetic ions in ITER will be different with respect to the JET DTE1 campaign of 1997, namely due to the different ratio between the width of the $\alpha$ s orbit and the machine size $(\sim 1 / 3$ during DTE1 in JET compared with $\sim 1 / 6$ to $\sim 1 / 10$ as expected for ITER), and between the birth energy of the $\alpha$ s and $E_{\alpha \mathrm{CRIT}}(\sim 30$ during DTE1 in JET compared with $\sim 15$ to $\sim 20$ as expected for ITER). Such differences mean that the micro-turbulent spectrum will importantly interact with Alfvén turbulence in ITER, thus affecting the overall turbulent transport $[68,69]$. Hence, timedependent nonlinear simulations will be needed to study and possibly optimize the various ITER operational scenarios and improve the predictions for the efficiency of this mechanism for ITG turbulence stabilization in ITER.

Regarding possible future DT experiments in JET, it would clearly be very useful if the operational scenario of the 1997 alpha-heating experiment could be repeated to provide further insights into the phenomenology presented here using the much advanced diagnostic capabilities for fast ions currently available, which are now capable of providing high precision measurements of the energy and pitch-angle distribution of the fusion-born $\alpha \mathrm{s}$. Further improvements would be required on the turbulence diagnostics in order to obtain reliable and high precision direct measurements of the time evolution of the amplitude and radial structure of the turbulence spectra. These data would be needed to provide further useful input for dedicated simulations aimed at providing a fully self-consistent and quantitative explanation of the effect of the $\alpha$ s on the suppression of ITG modes analysed here. In addition to this experimental work, it is clear that quantitative predictions on the ion temperature evolution that could go beyond the qualitative phenomenology that we have presented here can only be obtained if one is able to 
formally express the ion thermal conductivity $\chi_{\mathrm{i}}(r)$ directly not only as a function of the growth rate $\left(\gamma_{\mathrm{ITG}} / k_{y}^{2}\right)$ but also of the amplitude of the turbulent micro-instability fields $\Phi$, i.e. $\chi_{\mathrm{i}}(r)=\chi_{\mathrm{i}}\left(\gamma_{\mathrm{ITG}}(r), \Phi(\omega, n, r)\right)$, with $\gamma_{\mathrm{ITG}}(r)$ calculated by state-of-the-art codes such as GENE and $\Phi$ being any of the measured turbulence fields $\delta B_{\text {MEAS }}(\omega, n, r), \Delta T_{\text {MEAS }}(\omega, n, r)$ and $\Delta n_{\text {MEAS }}(\omega, n, r)$. This capability is not yet present in state-of-the-art transport codes, but it is felt that this could contribute to major advances in the physical understanding of the underlying turbulent transport mechanisms driven by fusion-born $\alpha \mathrm{s}$, which is likely to become a key issue in future burning plasma experiments such as ITER.

\section{Acknowledgments}

This work was partly supported by EURATOM under the contract of Association with CRPP-EPFL. This work was also partly supported by the Swiss National Science Foundation. The authors would like to acknowledge useful discussions with S. Brunner, S. Coda, A. Fasoli, J. Graves, J.B. Lister, L. Villard and D. Wagner (all at CRPP), P.R. Thomas at the ITER organization, and L. Garzotti and P.C. deVries at the JET organization. The authors would also like to thank the reviewers and O. Sauter (CRPP) for their comments and suggestions to the first draft of this paper.

\section{Appendix A. The sparse representation method and the SparSpec code}

Sparse representations $[7,70,71]$ (SRs) are mathematical models that account for all the information contained in an input data set with a linear combination of elementary signals called atoms. Among all the possible combinations of atoms, the one with the smallest number of atoms is the sparse representation of the signal. The SRS problem consists of minimizing the criterion:

$$
J_{0}(x)=\|\boldsymbol{y}-W \boldsymbol{x}\|^{2}+\gamma\|\boldsymbol{x}\|_{0} .
$$

Here $\boldsymbol{y}=\left[y_{1}, y_{2}, \ldots, y_{P}\right]$ is the vector of (noisy) input data taken at position $\phi_{p}, p=\{1, \ldots, P\}$ being the suffix labelling the individual sensors used for the measurement; $\boldsymbol{x}=\left[x_{1}, x_{2}, \ldots, x_{M}\right]$ is the vector of complex amplitudes used to model the input data $y, m==\{1, \ldots, M\}$ being the suffix labelling the individual atoms; $\boldsymbol{W}=\left[\boldsymbol{w}_{1}, \boldsymbol{w}_{2}, \ldots, \boldsymbol{w}_{M}\right]$ is the spectral window matrix, where the vector $\boldsymbol{w}_{k}$ corresponds to the $k$ th atom, the L0-norm of $\boldsymbol{x}$ : $\|\boldsymbol{x}\|_{0}=\#\left\{k,\left|x_{k}\right| \neq 0\right\}$ is the number of non-zero components of $\boldsymbol{x}$ and $\gamma$ is a penalization parameter. For the case of the turbulence spectra analysed in this work in the FFT time versus (angular) frequency $(\omega)$ and in the toroidal angle $(\phi)$ versus toroidal mode number $(n)$ domains, it is convenient to mathematically express each input data $y_{p}$ as

$$
y_{p}(\omega)=\sum_{l=1}^{L} A_{l}(\omega) \mathrm{e}^{\mathrm{i} n_{l} \phi_{p}}+\varepsilon_{p}(\omega)
$$

where $n_{l}$ and $A_{l}$ are the unknown mode numbers and complex amplitudes, respectively, $L \leqslant M$ is the unknown number of modes (i.e. the data are modelled using only a subset of the components that can be conceivably present in the input spectrum) and $\varepsilon_{p}$ corresponds to the (complex-valued) noise on the measurement made at the given $p$ th sensor.

To minimize the criterion of equation (A1), one must sift through all possible combinations of elementary atoms belonging to the chosen set of base functions, as one would in principle have to do when using the SVD methods. This approach becomes clearly and very quickly numerically intractable for large $L$ (as $M$ has to increase correspondingly to take full advantage of the SRS algorithm), particularly for the usual case of tokamak plasma physics where there is uneven spatial sampling, so that Nyquist-based criteria cannot be used. The only numerically efficient method to solve accurately this computational problem is to provide an estimate for the amplitudes of all possible $x_{k}$ components in the range $k=\{-K, \ldots, K\}$ (where $K$ is much larger than $M>L$, i.e. the atom basis set is much larger than the maximum number of components that can be conceivably present in the input spectrum), but at the same time enforcing that most of these modes have actually a null amplitude $x_{k}=0$. For numerical efficiency, we then replace the L0-norm (the number of the non-zero atoms representing the input data) with the L1norm (i.e. the sum of the amplitudes of the non-zero atoms representing the input data), i.e. using the so-called convex relaxation scheme (see, for instance, $[7,70,71]$ ). This leads to the new criterion:

$J_{0}(x)=\|\boldsymbol{y}-W \boldsymbol{x}\|^{2}+\lambda\|\boldsymbol{x}\|_{1}=\|\boldsymbol{y}-W \boldsymbol{x}\|^{2}+\lambda \sum_{k=-K}^{K}\left(\left|x_{k}\right|\right)$.

The penalization parameter $\lambda$ is related to the noise level and requires an appropriate tuning, since it increases the penalty for those solutions which invoke a larger number of modes. It can be shown that (a) for $\lambda>\lambda_{\mathrm{MAX}}=\max \left(\left|W^{H} y\right|\right)$, the minimizer $\boldsymbol{x}_{\mathrm{MIN}}$ of equation (A3) is identically zero, i.e. the unique solution has no detected modes; and (b) for any given $\lambda$, the minimizer $x_{\text {MIN }}$ of equation (A3) satisfies $\max \left(\mid W^{H}(y-\right.$ $\left.\left.W x_{\text {MIN }}\right) \mid\right) \leqslant \lambda$. Hence $\lambda$ can be interpreted as the maximum peak amplitude allowed in the periodogram of the residual (i.e. the data minus the model corresponding to the sum of the estimated modes), and choosing $\lambda$ to be a fraction $\lambda_{\text {NORM }} \in$ $[0 \rightarrow 1]$ of the maximum of the periodogram of the data $\lambda=$ $\lambda_{\text {NORM }} \times \max \left(\left|\boldsymbol{W}^{H} \boldsymbol{y}\right|\right)$ ensures the periodogram of the residual to be lower up to this fraction relatively to the maximum of the data periodogram. Hence, knowledge of the noise level in the measurements helps one to determine the optimum value for $\lambda_{\text {NORM }}$ to be used for the analysis of the turbulence data. As an example of this efficient tuning of $\lambda_{\text {NORM }}$, this quantity is set in the range $\lambda_{\mathrm{NORM}}=0.10$ to $\lambda_{\mathrm{NORM}}=0.35$ when performing the analysis of the very long time series typical of astronomical and astrophysical measurements [7], then $\lambda_{\mathrm{NORM}}=0.85$ to $\lambda_{\mathrm{NORM}}=0.95$ when performing in real-time the toroidal mode number decomposition of the frequencydegenerate spectrum of coherent AEs actively driven by an in-vessel antenna system in JET [52,72], so that only the largest component is selected for real-time tracking [73, 74], then $\lambda_{\text {NORM }}=0.35$ to $\lambda_{\text {NORM }}=0.95$ when the same analysis is performed post-pulse, so that effectively all (or a selection of the largest between) the coherent, antenna-driven AEs whose amplitude is well above the background turbulence level are 
found [39,73-77], and finally in the range $\lambda_{\text {NORM }}=0.03$ to $\lambda_{\text {NORM }}=0.10$ for the analysis reported here, where we indeed need to look into the background incoherent turbulence spectrum which sits well above the bit-noise level that is found in the data acquisition system in the absence of any plasma.

It is intuitive to see that the criterion of equation (A3) can be minimized more efficiently than that of equation (A1). However, this new criterion is not strictly convex, as the number of unknowns may be larger than the number of data, hence the uniqueness of the solution cannot be guaranteed a priori. Theoretical conditions guaranteeing the equivalence of both solutions have been established [7, 70, 71], which are based on properties of the spectral matrix $\boldsymbol{W}$, so depend on the specificities of the problem being considered. Furthermore, it has been shown from simulations and analysis of unevenly sampled measurements using comparison between different numerical methods that such a L1-norm penalized solution generally gives very satisfactory results in terms of detection, even in the case of multiple modes (see, for instance, [7, 39, 41, 72-79]).

The SRS method we use in the work presented here has been derived from the implementation of the SparSpec code (freeware available at: http://www.ast.obs-mip.fr/Softwares), which was originally developed for the analysis of astronomical data. The application of the SparSpec code to the real-time and post-pulse analysis of the JET measurements of various magneto-hydrodynamic modes, specifically AEs, has been previously presented elsewhere $[39,72-79]$ and the readers are referred to these publications for further details on this topic.

\section{References}

[1] Keilhacker M. for the JET team 1999 Nucl. Fusion 39209

[2] Thomas P.R. for the JET team 1999 Nucl. Fusion 391619

[3] Thomas P.R. et al 1998 Phys. Rev. Lett. 805548

[4] Thomas P.R. et al 2001 Alpha-heating of thermal ions in JET Proc. 28th EPS Conf. (Madeira, Portugal, 18-22 June 2001) vol 25A (ECA) Paper P3.001 http://www.cfn.ist.utl.pt/EPS2001/fin/pdf/P3.001.pdf

[5] Sips A.C.C. et al 2011 Scientific preparation for future D-T campaigns at JET in support of ITER Proc. 31st EPS Conf. (Strasbourg, France, 27 June-3 July 2011) vol 35G (ECA) Paper O5.127 http://ocs.ciemat.es/EPS2011PAP/ html/author.html

[6] Shimada M. et al 2007 Progress in the ITER Physics Basis: chapter 1: overview and summary Nucl. Fusion $47 \mathrm{~S} 1$

[7] Bourguignon S., Carfantan H. and Böhm T. 2007 Astron. Astrophys. 462379

[8] Jenko F. et al 2000 Phys. Plasmas 71904

[9] Testa D. and Albergante M. 2011 ITPA Energetic Particles Meeting (Austin, USA, 12-13 September 2011) http://w3 fusion.ph.utexas.edu/ifs/itpa/index.html\#program

[10] Testa D. and Albergante M. 2012 Europhys. Lett. 9735003

[11] Rimini F. for the JET team 1999 Nucl. Fusion 392025

[12] Sivukhin D.V. 1966 Reviews of Plasma Physics vol 4, ed M.A. Leontovich (New York: Consultants Bureau)

[13] Goldston R. et al 1981 J. Comput. Phys. 4361

[14] Cenacchi G. and Taroni A. 1988 JETTO: a free-boundary plasma transport code Report ENEA-RT-T113-88-5 Centro Ricerche Energia, Bologna, Italy

[15] Collection 1999 Nucl. Fusion 39 (collection devoted to the JET DTE1 experiments)

[16] The JET Team (prepared by V.V. Parail) 1999 Nucl. Fusion. 391743
[17] Wesson J. 2003 Tokamaks (3rd edn) (Oxford: Oxford Science Publication) pp 597-616

[18] Zweben S.J. et al for the TFTR team 2000 Nucl. Fusion 4091

[19] Scott S.D. et al 1995 Phys. Scr. 51394

[20] Hawryluk R.J. et al 1994 Phys. Rev. Lett. 723530

[21] Scott S.D. et al 1995 Phys. Plasmas 22299

[22] Budny R.V. 1994 Nucl. Fusion 341247

[23] Strachan J.D. 1997 Plasma Phys. Control. Fusion 39 B103

[24] Korotkov A.A., Gondhalekar A. and Akers R.J. 1998 Rep. JET-P(98)25 JET Joint Undertaking

[25] Korotkov A.A., Gondhalekar A. and Akers R.J. 2000 Phys. Plasmas 8957

[26] Testa D. and Gondhalekar A. 2000 Nucl. Fusion 40975

[27] Estrada-Mila C., Candy J. and Waltz R.E. 2006 Phys. Plasmas 13112303

[28] Angioni C. and Peeters A.G. 2008 Phys. Plasmas 15052307

[29] Garbet X. et al 2004 Plasma Phys. Control. Fusion 46 B557

[30] Waltz R.E. and Miller R.L. 1999 Phys. Plasmas 64265

[31] Lapillonne X. et al 2009 Phys. Plasmas 16032308

[32] Merz F. and Jenko F. 2010 Nucl. Fusion 50054005

[33] Albergante M., Graves J.P., Fasoli A. and Lapillonne X. 2010 Nucl. Fusion 50084013

[34] Weiland J. 2000 Collective Modes in Inhomogeneous Plasmas: Kinetic and Advanced Fluid Theory (Bristol: Institute of Physics Publishing)

[35] Chen Y. et al 2003 Nucl. Fusion 431121

[36] Fisch N.J. and Herrmann M.C. 1999 Plasma Phys. Control. Fusion 41 A221

[37] Blackler K. and Edwards A.W. 1994 IEEE Trans. Nucl. Sci. 41111

[38] Heeter R.F. et al 2000 Rev. Sci. Instrum. 714092

[39] Klein A. et al 2008 Plasma Phys. Control. Fusion 50125005

[40] Testa D., Fasoli A. and Solano E. 2003 Rev. Sci. Instrum. 741694

[41] Testa D. et al 2010 Fusion Sci. Technol. 57 208-37

[42] Akhiezer A.I. et al 1975 Plasma Electrodynamics vol 2 (Oxford: Pergamon)

[43] Piliya A.D. and Popov A.Yu. 2002 Plasma Phys. Control. Fusion $\mathbf{4 4} 2051$

[44] Bornatici M., Cano R., deBarbieri O. and Engelmann F. 1983 Nucl. Fusion 231153

[45] Parker S.E., Chen Y., Wan W., Cohen B.I. and Nevins W.M. 2004 Phys. Plasmas 112594

[46] Pueschel M.J., Kammerer M. and Jenko F. 2008 Phys. Plasmas 15102310

[47] Pueschel M.J. and Jenko F. 2010 Phys. Plasmas 17062307

[48] Bottino A. et al 2010 IEEE Trans. Plasma Sci. 382129

[49] Bottino A. et al 2011 Plasma Phys. Control. Fusion 53124027

[50] Scott B. 2003 Plasma Phys. Control. Fusion 45 A385

[51] Scott B. 2007 Plasma Phys. Control. Fusion 49 S25

[52] Fasoli A. et al 1995 Phys. Rev Lett. 75645

[53] Tardini G. et al 2007 Nucl. Fusion 47280

[54] Thomas P.R. 2011 private communication, ITER organization

[55] Rebut P.H. for the JET team 1992 Plasma Phys. Control. Fusion 341749

[56] Jacquinot J. for the JET team 1999 Nucl. Fusion 39235

[57] Gormezano C. for the JET team 1998 Phys. Rev. Lett. 805544

[58] Biglari H., Diamond P.H. and Rosenbluth M.N. 1989 Phys. Fluids B1 109

[59] Wesson J. 2003 Tokamaks 3rd edn (Oxford: Oxford Science Publication) pp 428-9

[60] Glanz J. 1996 Science 2741600

[61] Carreras B.A. 1997 Nature 385300

[62] Macilwain C. 1997 Nature 388115

[63] Moyer M. 2010 Scientific American pp 50-7 March 2010

[64] Gormezano C. et al 2007 Progress in the ITER Physics Basis: chapter 6: steady state operation Nucl. Fusion 47 S285

[65] Albergante M. et al 2011 Plasma Phys. Control. Fusion 53054002

[66] Pereverzev G. et al 1991 Max-Planck-Institut für Plasmaphysik Report IPP 5/42 (Garching, Germany) 
[67] Gribov Y. et al 2007 Progress in the ITER Physics Basis: chapter 8: plasma operation and control Nucl. Fusion 47 S385

[68] Bass E. and Waltz R.E. 2010 Phys. Plasmas 17112319

[69] Zonca F., Chen L., Dong J.Q. and Santoro R.A. 1999 Phys. Plasmas 61917

[70] Donoho D. 2006 J. Commun. Pure Appl. Math. 59797

[71] Bourguignon S., Carfantan H. and Idier J. 2007 IEEE J. Sel. Top. Signal Process. 14

[72] Testa D. et al 2004 The new Alfvén Wave Active Excitation System at JET Proc. 23rd SOFT Conference (Venice, Italy,
20-24 September 2004) http://infoscience.epfl.ch/record/ 143354/files/

[73] Testa D.et al 2011 Fusion Eng. Des. 86381

[74] Testa D. et al 2010 Europhys. Lett. 9250001

[75] Testa D. et al 2010 Nucl. Fusion 50084010

[76] Panis T. et al 2010 Nucl. Fusion 50084019

[77] Testa D. et al 2011 Nucl. Fusion 51043009

[78] Panis T., Fasoli A. and Testa D. 2012 Nucl. Fusion 52023013

[79] Panis T., Fasoli A. and Testa D. 2012 Nucl. Fusion 52023014 